\title{
Claudins in Renal Physiology and Pathology
}

\author{
Caroline Prot-Bertoye ${ }^{1,2,3,4,5}$ and Pascal Houillier 1,2,3,4,5,* \\ 1 Centre de Recherche des Cordeliers, INSERM, Sorbonne Université, Université de Paris, F-75006 Paris, \\ France; caroline.bertoye@aphp.fr \\ 2 Service de Physiologie, Hôpital Européen Georges Pompidou, Assistance Publique-Hôpitaux de Paris, \\ F-75015 Paris, France \\ 3 Centre de Référence des Maladies Rénales Héréditaires de l’Enfant et de l'Adulte (MARHEA), F-75015 Paris, \\ France \\ 4 Centre de Référence des Maladies Rares du Calcium et du Phosphate, F-75015 Paris, France \\ 5 CNRS, ERL8228, F-75006 Paris, France \\ * Correspondence: pascal.houillier@aphp.fr
}

Received: 15 January 2020; Accepted: 24 February 2020; Published: 10 March 2020 updates

\begin{abstract}
Claudins are integral proteins expressed at the tight junctions of epithelial and endothelial cells. In the mammalian kidney, every tubular segment express a specific set of claudins that give to that segment unique properties regarding permeability and selectivity of the paracellular pathway. So far, 3 claudins (10b, 16 and 19) have been causally traced to rare human syndromes: variants of CLDN10b cause HELIX syndrome and variants of CLDN16 or CLDN19 cause familial hypomagnesemia with hypercalciuria and nephrocalcinosis. The review summarizes our current knowledge on the physiology of mammalian tight junctions and paracellular ion transport, as well as on the role of the 3 above-mentioned claudins in health and disease. Claudin 14, although not having been causally linked to any rare renal disease, is also considered, because available evidence suggests that it may interact with claudin 16 . Some single-nucleotide polymorphisms of CLDN14 are associated with urinary calcium excretion and/or kidney stones. For each claudin considered, the pattern of expression, the function and the human syndrome caused by pathogenic variants are described.
\end{abstract}

Keywords: claudin 10b; claudin 16; claudin 19; claudin 14; kidney; tight junction; HELIX syndrome; familial hypomagnesemia with hypercalciuria and nephrocalcinosis; sodium; divalent cations

\section{Introduction}

Almost every epithelium in multicellular organisms separates two compartments (apical and basolateral) with differing ion composition. Depending on the nature of the compartments, human epithelia can behave as barriers (e.g., the skin epithelium) or allow selective transport of solutes and/or solvent from apical to basolateral compartment or vice-versa (e.g., intestinal or renal tubular epithelia). The asymmetry of composition between apical and basolateral compartment is created and maintained by active transport (with the notable exception of passive transport that occurs as a result of Gibbs-Donnan effect). Active transport can occur transcellularly only, at the expense of energy release; for example hydrolysis of ATP releases energy used to actively pump ions across the plasma membrane and to build transmembrane electrochemical gradients. Passive diffusion between 2 compartments may be defined as the flow of solute that occurs in response to the difference in electrochemical potentials of the considered solute between both compartments. In an epithelium, diffusion can occur across plasma membranes or along the paracellular pathway. Because of the existing transmembrane electrochemical potential differences, sodium $\left(\mathrm{Na}^{+}\right)$, calcium $\left(\mathrm{Ca}^{2+}\right)$ and magnesium $\left(\mathrm{Mg}^{2+}\right)$ cannot cross passively both apical and basolateral membranes: therefore, at some step, transcellular transport of $\mathrm{Na}^{+}, \mathrm{Ca}^{2+}$ 
and $\mathrm{Mg}^{2+}$ requires energy expenditure. By contrast, passive paracellular diffusion of $\mathrm{Na}^{+}, \mathrm{Ca}^{2+}$ and $\mathrm{Mg}^{2+}$ allows both transepithelial transport and energy saving [1]. The present review is a summary of our knowledge of normal and abnormal paracellular ion transport in the mammalian renal tubule. Specifically, it focuses on the role of claudin proteins, which are highly specialized proteins expressed at the tight junction, in health and disease.

\section{Structure and Function of Tight Junction in the Kidney}

The properties of the paracellular pathway along the renal tubular epithelium have been less intensively studied than the properties of the transcellular pathway. Nevertheless, epithelia have been classified as leaky or tight, according to the value of transepithelial resistance, $\mathrm{R}_{\mathrm{T}}$. Because transcellular and paracellular pathways are organized in parallel, the reciprocal of transepithelial resistance equals the sum of the reciprocal of transcellular resistance $\left(R_{C}\right)$ and of the reciprocal of paracellular resistance $\left(\mathrm{R}_{\mathrm{P}}\right)$, according to Ohm's law,

$$
\frac{1}{\mathrm{R}_{\mathrm{T}}}=\frac{1}{\mathrm{R}_{\mathrm{C}}}+\frac{1}{\mathrm{R}_{\mathrm{P}}}
$$

The direct consequence, assuming that $R_{C}$ is high and almost constant, is that «leaky» epithelia (low $R_{T}$ ) must have low $R_{P}$ and «tight» epithelia (high $R_{T}$ ) have high $R_{P}$. Several investigators have measured $R_{T}$ in the various segments of the renal tubule of rodents and rabbits; they consistently found that the proximal tubule has the lowest $R_{T}$ of all segments and that $R_{T}$ increases to a maximal value in the inner medullary collecting duct (for review, see Reference [2]). Besides electrical resistance, every paracellular pathway is also characterized by its selectivity to ion charge and size [3-5]. The size selectivity of paracellular pathways was studied by several investigators, mostly in cells lines or intestinal epithelia. Most studies reported that at least two populations of pores are present in the paracellular pathway, the most restrictive having a pore size of 4-8 $\AA$ [6-10]. The diameter of the major cations in extracellular fluid is 6.62-8.60 $\AA$ when they are in their hydrated form and 1.44-2.98 when they are in their unhydrated form [11]. The selectivity to ion charge vary from epithelium to epithelium: some are cation selective whereas other are anion selective. Most of the resistance and charge selectivity of the paracellular pathway are determined at the tight junction. Tight junction is formed by a complex of multiple proteins, subdivided into transmembrane proteins and cytoplasmic plaque proteins, including signaling proteins and scaffolding proteins (adapters that link the tight junction complex to the actin cytoskeleton) [12,13]; tight junction contacts and their selective permeation properties are created by a large family of transmembrane proteins called claudins [14-16]. In mammals, the claudin gene family is composed of at least 27 members $[2,17,18]$. Claudins are relatively short transmembrane proteins that possess between 207 and 305 amino acids. The calculated molecular mass is between 21 and $34 \mathrm{kDa}$. The general structure of all claudins is similar, consisting in a short intracellular $\mathrm{NH}_{2}$ terminus, a longer intracellular $\mathrm{COOH}$ terminus, two extracellular segments (ECS1 and ECS2) (which form a $\beta$-sheet of five $\beta$-strands ( $4 \beta$-strands in ECS1 and $1 \beta$-strand in ECS2)), one intracellular loop and four transmembrane domains (helices). The first extracellular segment ECS1 contains the common motif W-LW-C-C and positively and negatively charged amino acid residues that determine the charge selectivity. This motif is critical for folding ECS1 in a region close to the plasma membrane and to form the characteristic $\beta$-sheet fold of ECS1 and ECS2 [19]. Claudins polymerize in tight junctions strands through cis (between two claudin within the same cell membrane) and trans (between claudins on opposing cell membranes) interactions [17] (Cis-polymerization would involve a short extracellular helix (ECH) at the end of ECS1 and the extracellular extension of the third transmembrane domain TM3 of a neighboring claudin [17]. Trans-interaction of claudins between neighboring cells is presumed to involve the intercellular interaction of the variable regions V1 (in ECS1) and V2 (in ECS2) $[17,20,21]$. An hypothetical model has been proposed: tight junction strands would consist of anti-parallel double-rows of claudins and the paracellular channel would be achieved by the interaction of the four claudins coming from double-rows [22]. A trans-interacting octamer model with a double pore has also been proposed $[21,23])$. 
Among the claudin family, a highly conserved PDZ -binding domain is present near the end of the carboxy-terminal end, which binds to the homologous domain of scaffolding proteins such as ZO-1, which is important for the localization of claudins at the tight junction $[24,25]$. The $\mathrm{COOH}$ terminus also contains a number of potential phosphorylation sites, reported to play a role in the localization of claudins at the tight junction.

Many claudins are expressed along the mammalian renal tubule and collecting duct. Although some controversies remain regarding the exact localization of a specific claudin, the overall pattern is that every tubular segment expresses a specific association of claudins, likely accounting for the specific properties of the paracellular pathway of every nephron segment (Figure 1).

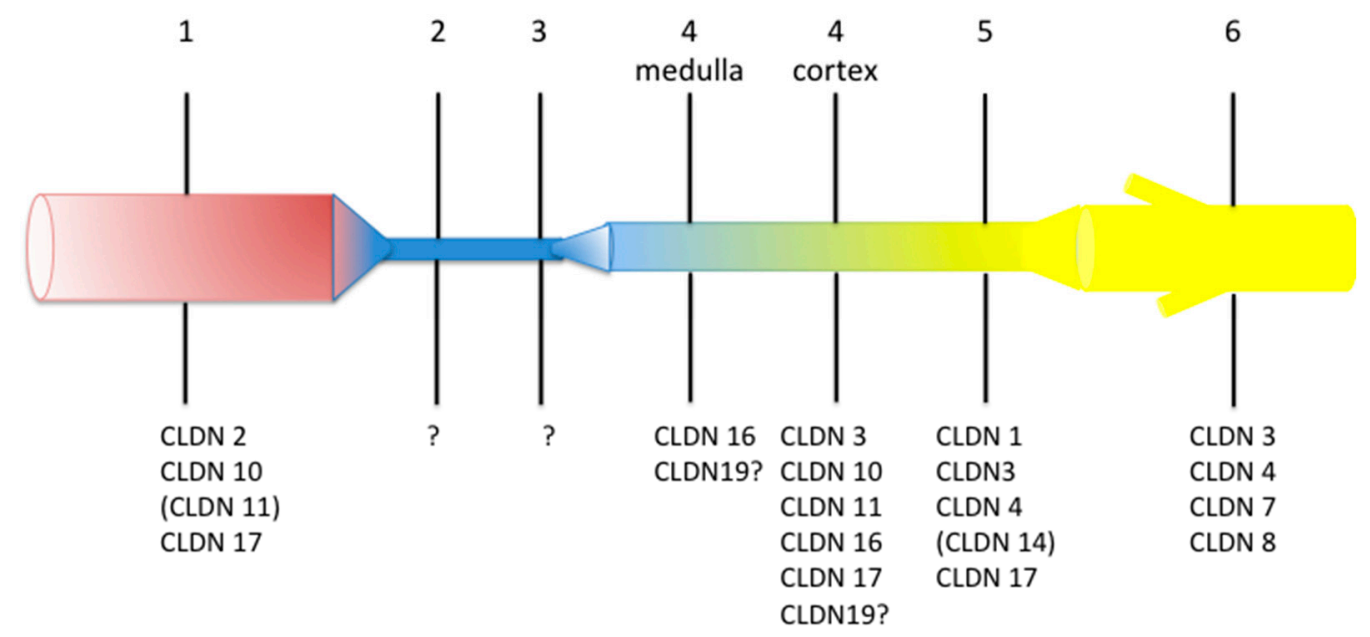

Figure 1. Pattern of expression of claudin (CLDN) proteins along the human renal tubule and collecting duct. The figure is a summary of data published in References [26-29] (the expression pattern in rodent kidney has recently been summarized in several reviews [2,30,31]). Question mark means that direct evidence of the expression of the considered claudin in human is not available but that indirect evidence suggests that it should be expressed there. Brackets mean that the expression is low. The numbers above the various segments are: 1 , proximal convoluted and straight tubule; 2 , descending thin limb; 3 , ascending thin limb; 4, distal straight tubule (thick ascending limb); 5, distal convoluted tubule; 6 , cortical and outer medullary collecting duct. Colors illustrate the fact that composition and/or volume of tubular fluid changes along the renal tubule. Readers interested in mRNA expression pattern can visit https://hpcwebapps.cit.nih.gov/ESBL/Database/NephronRNAseq/All_transcripts.html.

So far, only a few claudins have been causally involved in human monogenic diseases; they are claudin (CLDN) 10b, CLDN16 and CLDN19 and will be considered below.

\section{Claudin $10 \mathrm{~b}$ and the HELIX Syndrome}

The human CLDN10 gene is located on chromosome 13q31-q34 and contains five exons [32]. There are two claudin 10 splice variants differing in the first exon that encode two main claudin 10 isoforms, claudin 10a and claudin 10b [33].

\subsection{Phenotype}

In 2017, three independent groups described the clinical consequences of biallelic mutations of the CLDN10 gene.

The first report described the phenotype of two unrelated patients bearing compound heterozygous mutations (Table 1, [34]). One adult female patient had hypokalemic metabolic alkalosis, chronic kidney disease (CKD) stage 3, polyuria, low urinary $\mathrm{Mg}^{2+}$ excretion and a tendency toward hypermagnesemia; the second patient displayed similar abnormalities associating hypokalemic metabolic alkalosis and a tendency toward hypermagnesemia. The second report described the phenotype of 13 affected 
patients from 2 distantly related families, all carrying the homozygous missense mutation in exon 1b (Table 1, [35]). All affected patients had anhidrosis, heat intolerance, inability to produce tears (alacrimia) and xerostomia. A biological assessment was available in 6 among the 13 patients, aged 23-47 years. All had normal plasma potassium concentration and plasma $\mathrm{Mg}^{2+}$ level was either high or in the upper range of normal values. Estimated glomerular filtration rate (eGFR) was higher than $60 \mathrm{~mL} / \mathrm{min} / 1.73 \mathrm{~m}^{2}$. Plasma renin and aldosterone concentrations were not available. The third report described the phenotype of 6 patients from 2 unrelated families [28]. All patients were bearing one homozygous missense mutation of the CLDN10 gene. All patients had hypohidrosis, renal loss of $\mathrm{NaCl}$ with secondary hyperaldosteronism and hypokalemia, as well as hypolacrimia, ichthyosis, xerostomia and severe enamel wear. Biological assessment showed plasma $\mathrm{Mg}^{2+}$ levels either high or at the upper limit of reference range and a blunted natriuretic and chloruretic response to furosemide infusion. The watery component of saliva was severely reduced in patients. Heterozygous family members were asymptomatic. Finally, the most recent report to date describes a teenager carrying a biallelic mutation of CLDN10 [36]. The patient had anhidrosis, xerostomia, alacrimia and hypokalemia; he developed hypermagnesemia and CKD over a 4 years follow-up period. The acronym HELIX (Hypohidrosis, Electrolyte disturbances, hypoLacrimia, Ichthyosis, Xerostomia) has been coined to name this syndrome (Online Mendelian Inheritance in Man (OMIM) \#617671).

Table 1. Similarities and differences among phenotype in patients with HELIX syndrome.

\begin{tabular}{|c|c|c|c|c|c|}
\hline Reference & $\begin{array}{c}\text { Bongers } \\
\text { [34] }\end{array}$ & Klar [35] & $\begin{array}{c}\text { Hadj-Rabia } \\
\text { [28] }\end{array}$ & Meyers [36] & Overall (\%) \\
\hline Area of Origin & Europe & Pakistan & $\begin{array}{l}\text { North Africa, } \\
\text { Pakistan }\end{array}$ & South America & \\
\hline Consanguinity & No & Yes & Yes & Yes & \\
\hline Hypohidrosis & N.D. & $13 / 13$ & $6 / 6$ & $1 / 1$ & $20 / 20(100 \%)$ \\
\hline Electrolyte imbalance & $2 / 2$ & $6 / 7$ & $6 / 6$ & $1 / 1$ & $15 / 16(94 \%)$ \\
\hline Hypolacrimia & N.D. & $13 / 13$ & $6 / 6$ & $1 / 1$ & $20 / 20(100 \%)$ \\
\hline Ichthyosis & N.D. & N.D. & $6 / 6$ & $0 / 1$ & $6 / 7(86 \%)$ \\
\hline Xerostomia & N.D. & $13 / 13$ & $6 / 6$ & $1 / 1$ & $20 / 20(100 \%)$ \\
\hline \multicolumn{6}{|l|}{ Plasma abnormalities } \\
\hline Hypokalemia & $2 / 2$ & $0 / 7$ & $3 / 6$ & $1 / 1$ & $6 / 16(38 \%)$ \\
\hline Hypermagnesemia & $1 / 2$ & $6 / 7$ & $6 / 6$ & $1 / 1$ & $14 / 16(88 \%)$ \\
\hline $\begin{array}{l}\mathrm{eGFR}<60 \\
\mathrm{~mL} / \mathrm{min} / 1.73 \mathrm{~m}^{2}\end{array}$ & $1 / 2$ & $0 / 3$ & $1 / 6$ & $1 / 1$ & $3 / 12(25 \%)$ \\
\hline $\begin{array}{l}\text { Secondary } \\
\text { hyperaldosteronism }\end{array}$ & N.D. & N.D. & $6 / 6$ & $\begin{array}{c}\text { Hyperaldosteronism } \\
\text { without } \\
\text { hyperreninism }\end{array}$ & \\
\hline Nephrolithiasis & $0 / 2$ & $4 / 13$ & $0 / 6$ & $0 / 1$ & $4 / 22(18 \%)$ \\
\hline
\end{tabular}

N.D.: not determined.

The similarities and differences between the various reports are summarized in Table 1 . All patients with the HELIX syndrome have a functional defect of sweat, salivary and lacrimal glands; most of them have blood electrolyte abnormalities, hypermagnesemia and/or hypokalemia; $25 \%$ have CKD stage 3 and none has nephrocalcinosis.

\subsection{Variants/Pathogenesis}

Seven pathogenic, or likely to be pathogenic, nucleotide abnormalities have been reported in patients with HELIX syndrome, so far (Table 2). Three of them are located into exon $1 \mathrm{~b}$ and affect exclusively the protein CLDN10b; 4 are into exon 2, 3 or 4 and affect both CLDN10a and CLDN10b protein. However, no obvious difference can be seen between the phenotypes of patients bearing only mutated CLDN10b and patients carrying mutated CLDN10a and CLDN10b, which does not help to understand the specific role of the former. 
Table 2. CLDN10 disease-causing variants [32].

\begin{tabular}{ccccccc}
\hline \multicolumn{7}{c}{ Missense/Nonsense Mutations } \\
\hline Ref. & $\begin{array}{c}\text { Nucleotide } \\
\text { Change }\end{array}$ & $\begin{array}{c}\text { Amino Acid } \\
\text { Change }\end{array}$ & $\begin{array}{c}\text { Protein } \\
\text { Change }\end{array}$ & $\begin{array}{c}\text { Variant } \\
\text { Class }\end{array}$ & Exon & Domain \\
\hline$[28]$ & c.2T $>$ C & Met1Thr & p.M1? & DM & $1 \mathrm{~b}$ & Helical \\
{$[35]$} & c.144C $>$ G & Asn48Lys & p.N48K & DM & $1 \mathrm{~b}$ & ECS1 \\
{$[34]$} & c.217G $>$ A & Asp73Asn & p.D73N & DM? & $1 \mathrm{~b}$ & ECS1 \\
{$[36]$} & c.238A $>$ G & Arg80Gly & p.R80G & DM & 2 & ECS1 \\
{$[28]$} & c.386C $>$ T & Ser131Leu & p.S131L & DM & 3 & Helical \\
{$[34]$} & c.446C $>$ G & Pro149Arg & p.P149R & DM? & 3 & ECS2 \\
\hline & & \multicolumn{5}{c}{ Splicing Mutations } \\
\hline$[34]$ & c.465-1G $>$ A & p.E157_T192del & DM? & 4 & Helical \\
\hline
\end{tabular}

Variant class is described according the Human Gene Mutation Database [37] DM: Disease-causing mutations; DM?: probable/possible pathological mutation; ECS1: first extracellular segment; ECS2: second extracellular segment.

The regular pattern of expression of claudin 10 isoforms in the mammalian kidney is a matter of controversy. In in situ hybridization experiments, Van Itallie and coworkers showed that Cldn10a transcripts were predominantly expressed by cortical tubules, whereas Cldn $10 \mathrm{~b}$ transcripts were predominantly expressed in tubular segments located in the outer medulla [33]. A more detailed pattern was reported by Günzel and collaborators [38]—-they reported that Cldn10a transcripts were expressed in the proximal convoluted tubule, cortical thick ascending limb (C-TAL) and cortical collecting duct, contrasting with $C l d n 10 b$ transcripts tubular expression that was restricted to the medullary thick ascending limb (M-TAL) and inner and outer medullary collecting duct. Hadj-Rabia et al. and Breiderhof et al. reported partially different results—Cldn10a transcripts were exclusively found in convoluted and straight proximal tubule and Cldn10b transcripts in the M- and C-TAL; none was significantly detectable in more distal segments [28,39].

No antibody is available that helps to distinguish between claudin $10 \mathrm{a}$ and claudin $10 \mathrm{~b}$ proteins. Van Itallie et al. reported that Cldn10 was strongly expressed at the tight junction of M- and C-TAL, thin ascending limb and medullary collecting duct and, to a lesser extent, at the tight junction of proximal tubule and cortical collecting duct [33].

Both in vitro (Table 3) and in vivo experiments have been conducted to unravel the function of Cldn 10b.

When heterologously expressed in MDCK-C7 or LLC-PK1 cells, both human and mouse claudin 10b decrease transepithelial resistance $[33,38,40]$; both human and mouse claudin $10 \mathrm{~b}$ increase permeability (P) ratios $\mathrm{P}_{\mathrm{Na}} / \mathrm{P}_{\mathrm{Cl}}, \mathrm{P}_{\mathrm{Mg}} / \mathrm{P}_{\mathrm{Cl}}$ and $\mathrm{P}_{\mathrm{Ca}} / \mathrm{P}_{\mathrm{Cl}}$ in MDCK-C7 cells [38]. By contrast, mouse Cldn10b affects neither transepithelial resistance nor $\mathrm{P}_{\mathrm{Na}} / \mathrm{P}_{\mathrm{Cl}}$, when expressed in MDCK-II cells that form a low resistance epithelial layer [33]. 
Table 3. Function of claudin $10 \mathrm{~b}$ according to heterologous expression studies in cell lines.

\begin{tabular}{|c|c|c|c|c|c|c|c|c|c|}
\hline Claudin & Cell Line & Transfection & TER & $\mathbf{P}_{\mathrm{Na}} / \mathbf{P}_{\mathrm{Cl}}$ & $P_{\mathrm{Na}}$ & $\mathbf{P}_{\mathrm{Cl}}$ & $\mathbf{P}_{\mathrm{Mg}} / \mathbf{P}_{\mathrm{Cl}}$ & $\mathbf{P}_{\mathrm{Ca}} / \mathbf{P}_{\mathrm{Cl}}$ & Ref. \\
\hline $\begin{array}{l}\text { Mouse } \\
\text { Cldn10b }\end{array}$ & MDCK II & Stable & NS & NS & NS & NS & & & [33] \\
\hline $\begin{array}{l}\text { Mouse } \\
\text { Cldn10b }\end{array}$ & LLC-PK1 & Stable & $\searrow$ & NS & $\nearrow$ & NS & & & [33] \\
\hline $\begin{array}{l}\text { Mouse } \\
\text { Cldn10b }\end{array}$ & MDCK-C7 & Stable & $\searrow$ & $\nearrow$ & & & $\nearrow$ & $\nearrow$ & [38] \\
\hline $\begin{array}{l}\text { Human } \\
\text { CLDN10b }\end{array}$ & MDCK-C7 & Stable & $\searrow$ & $\nearrow$ & & & $\nearrow$ & $\nearrow$ & [38] \\
\hline $\begin{array}{l}\text { Human } \\
\text { CLDN10b }\end{array}$ & MDCK-C7 & Stable & $\searrow$ & & & & & & [40] \\
\hline Mouse Cldn10a & MCDK II & Stable & NS & $\searrow$ & $\searrow$ & $\nearrow$ & & & [33] \\
\hline Mouse Cldn10a & LLC-PK1 & Stable & $\searrow$ & NS & NS & $\nearrow$ & & & [33] \\
\hline Mouse Cldn10a & MDCK II & Stable & NS & $\searrow$ & & & & & [38] \\
\hline Mouse Cldn10a & MDCK-C7 & Stable & NS & NS & & & NS & NS & [38] \\
\hline $\begin{array}{l}\text { Human } \\
\text { CLDN10a }\end{array}$ & MDCK-C7 & Stable & $\searrow$ & NS & & & NS & NS & [38] \\
\hline $\begin{array}{l}\text { Human } \\
\text { CLDN10a }\end{array}$ & MDCK-C7 & Stable & NS & & & & & & [40] \\
\hline
\end{tabular}

In electrophysiological studies, relative epithelial permeabilities (e.g., $\mathrm{P}_{\mathrm{Na}} / \mathrm{P}_{\mathrm{Cl}}$ ) are calculated using the Goldman-Hodgkin-Katz equation and the diffusion potential caused by the application of distinct solutions at the apical and basolateral compartment. Absolute permeabilities can be calculated if the transepithelial conductance is known using the Kimizuka-Koketsu equation [41]. The effects of claudin 10a have been included, for comparison with those of claudin 10b. TER: trans epithelial resistance; $\mathrm{P}_{\mathrm{X}}$ : permeability to ion $\mathrm{X}$; NS: not significantly different from control.

Inconsistent results on permeability to $\mathrm{Ca}^{2+}, \mathrm{Mg}^{2+}, \mathrm{Na}^{+}$and chloride may result from the endogenous expression of distinct claudins in the cell lines used in experiments. Overexpression of any claudin in a cell line with a claudin background different from that normally co-expressed with the claudin under investigation can induce variable/conflicting interaction and results [42].

Mice (10 weeks old) with a deletion of Cldn10b in the TAL (Cldn10b cKO) show marked hypermagnesemia with low fractional excretion of $\mathrm{Mg}^{2+}$ in urine, mild hyperphosphatemia, mild polyuria with inability to concentrate urine upon drinking water deprivation, elevated fractional excretion of potassium and medullary nephrocalcinosis without overt impairment in GFR [39]. Fractional excretion of $\mathrm{Na}^{+}$was not consistently elevated in $\mathrm{Cldn} 10 b \mathrm{cKO}$ mice. Noteworthy, most of the in vivo effects of $\mathrm{Cldn} 10 \mathrm{~b}$ deletion, except polyuria and high fractional excretion of potassium, were corrected by simultaneous deletion of Cldn16 (see FHHNC, below) [43].

Several of the mutant proteins have been heterologously expressed in various cell lines. In HEK293 and MDCK-C7 cells, cell surface expression of p.P149R and p.D73N CLDN10b was not decreased, as compared to wild-type protein, whereas that of p.E157_T192del CLDN10b, p.N48K CLDN10b or CLDN10b $\Delta 4$ (exon 4 was deleted) was reduced [34,35]. When expressed in MKTAL cells [44], both p.M1? and p.S131L mutated CLDN10b proteins were weakly expressed at cell surface, as compared with wild-type protein [28].

Isolated perfused TALs from $\mathrm{Cldn10b} \mathrm{cKO}$ mice had a higher transepithelial resistance and a lower paracellular permeability ratio $\mathrm{P}_{\mathrm{Na}} / \mathrm{P}_{\mathrm{Cl}}$ than TALs from normal littermates [39]. Based on the results described above, a consensual agreement is that the specific effect of claudin $10 \mathrm{~b}$ is to increase the paracellular permeability to $\mathrm{Na}^{+}$. Actually, a lower paracellular $\mathrm{P}_{\mathrm{Na}}$ in TAL, sweat glands and salivary glands may account for most of the clinical consequences of CLDN10b loss-of-function mutations. In the M-TAL, fifty per cent of $\mathrm{Na}^{+}$is passively reabsorbed along the paracellular pathway: a decrease in $\mathrm{P}_{\mathrm{Na}}$ impairs $\mathrm{Na}^{+}$reabsorption, causing a renal loss of $\mathrm{Na}^{+}$, extracellular fluid depletion with secondary hyperaldosteronism and a renal loss of potassium; the high transepithelial voltage would elevate passive paracellular $\mathrm{Mg}^{2+}$ and $\mathrm{Ca}^{2+}$ reabsorption in the TAL. In salivary and sweat ducts, where chloride is secreted transcellularly, $\mathrm{Na}^{+}$is passively secreted along the paracellular pathway. A loss-of-function mutation of $C L D N 10 b$ would decrease $\mathrm{Na}^{+}$secretion and the formation of saliva and sweat. 


\subsection{Prognosis and Treatment}

The long-term prognosis is unknown; however, 25\% of patients had CKD stage 3 at the time of presentation and close follow-up is advised.

No specific treatment is available to improve the condition of patients with HELIX syndrome. High $\mathrm{NaCl}$ and fluid intake is advised, with potassium supplements and drugs blocking aldosterone secretion and/or action when hypokalemia is present. Artificial tears and saliva can be used to relieve symptoms of eye and mouth dryness, respectively. Prolonged intense physical activity should be discouraged, particularly when the outside temperature is high, to prevent the risk of hyperthermia.

\section{Claudin 16, Claudin 19 and Familial Hypomagnesemia with Hypercalciuria and Nephrocalcinosis (FHHNC)}

Familial hypomagnesemia with hypercalciuria and nephrocalcinosis is an autosomal recessive disorder caused by variants of the CLDN16 (OMIM \#248250) and CLDN19 (OMIM \#248190) genes.

The human CLDN16 gene is located on chromosome 3q27 and contains five exons. Two potential start codons produce either a long ( 305 amino acids, $33 \mathrm{kDa}$ ) or short ( 235 amino acids, $27 \mathrm{kDa}$ ) CLDN16 protein isoform $[45,46]$. It is unknown which one of the two versions (long or short) is the physiologically relevant or whether both are functional. In vitro, Hou et al. described that the short CLDN16 is expressed at cell-cell borders whereas the long CLDN16 is targeted to endosomes and lysosomes [46]. However, in other studies, full length CLDN16 is expressed at the tight junction [47,48].

The human CLDN19 gene, located on chromosome 1p34.2, contains also fives exons, encoding CLDN19, a protein of 224 amino acids [32].

Because of the autosomal recessive pattern of inheritance, parental consanguinity is frequent [26,49]. The exact prevalence of FHHNC is unknown but it is a very rare disorder, likely to be under-diagnosed.

\subsection{Phenotype}

FHHNC is characterized by an excessive urinary loss of $\mathrm{Ca}^{2+}$ and $\mathrm{Mg}^{2+}$, resulting in hypomagnesemia and hypercalciuria. Patients have nephrocalcinosis (a parenchymal deposition of calcium-based crystal in the renal parenchyma) and renal failure that may progress toward end stage renal disease early in life [26,45,49-51]. Of note, hypomagnesemia can be absent [51,52].

Age at onset ranges from 0 to more to 30 years for CLDN16 [45,49,50,52-56] and CLDN19 mutations $[50,51,57,58]$ and the diagnosis can be delayed.

Patients commonly present with recurrent urinary tract infections, hematuria and abacterial leukocyturia, nephrolithiasis, polyuria/polydipsia with nycturia from infanthood [50-52,59-62] due to impaired urinary concentrating ability [54]. Hypocitraturia is frequently mentioned [45,52,54,55, 61,63-67] but urinary acidification capacity has seldom been assessed. A few reports mention distal defect of urinary acidification $[45,54,59]$ : incomplete distal renal tubular acidosis may affect up to $80 \%$ of patients $[45,66]$ but it remains unclear whether it is directly caused by CLDN16/19 mutation or by nephrocalcinosis. Hypocitraturia may also be linked to renal failure.

Parathyroid hormone levels seems to be higher than in control patients with chronic renal failure of other origin [49], which may be due to hypomagnesemia-related secondary hyperparathyroidism.

Patients with CLDN19 mutations frequently display severe ocular abnormalities (myopia, pigmentary retinitis, macular coloboma, strabismus, astigmatism, nystagmus, macular scars, macular degeneration, anisocoria, retinochoroiditis) $[50,51,57,58,68]$, contrasting with milder and rarer ocular abnormalities reported with CLDN16 mutations (strabismus, myopia, astigmatism, hypermetropia) [45]. CLDN19 is expressed in human fetal retinal pigment epithelium; claudin 19 may be involved in the development and the function of retinal pigment epithelium and retinal neurogenesis [69,70].

Cldn16 and Cldn19 are also expressed in ameloblast tight junction and loss-of-function mutations of CLDN16 and CLDN19 genes are associated with amelogenesis imperfecta [71,72]. The lack of Cldn16 strongly impairs tight junction organization in secretory stage ameloblasts [71].

An ophthalmological and a dental evaluation are required. 
Muscular-exercise intolerance with pain, weakness and electromyographical alterations [67] has been reported with CLDN19 mutation but whether this finding is specific of CLDN19 patients' needs to be confirmed: hypomagnesemia can lead to non-specific symptoms (muscle weakness and tetany [62]) but Cldn19 is also expressed in murine Schwann cells [73]. Cldn19-deficient mice exhibit behavioral abnormalities that could be due to peripheral neuropathy [73]. Accurate assessment of neuromuscular status in CLDN16 patients has to be performed in order not to miss subtle abnormalities.

Bone disease [74], bone deformities [62], failure to thrive [50-52,62,75] and rickets [45,52,62,76] are reported but their exact pathophysiological mechanisms need to be elucidated.

Histories of nephrolithiasis, nephrocalcinosis, and/or hypercalciuria can be observed in heterozygous family members not affected by FHHNC $[45,58,66,67,76]$ however other causes of these affections are not documented in cases reported.

\subsection{Prognosis and Treatment}

The prognosis of FHHNC is poor as it leads frequently to renal failure in childhood. All patients are affected by nephrocalcinosis but the severity of renal failure is variable.

In order to establish a genotype-phenotype correlation, Konrad et al. studied the function of CLDN16 mutants overexpressed in vitro and classified mutants according to a partial or a total loss of function: the age at onset of symptoms was younger and the decline of GFR was faster in patients bearing CLDN16 mutations causing a complete loss of function in both alleles [49].

However, there is a clinical heterogeneity of disease severity in families even among patients bearing the same CLDN16 [45,62,77,78] and CLDN 19 mutation [58].

Patients with CLDN19 mutations progress to chronic kidney disease (defined as an eGFR $<60 \mathrm{~mL} / \mathrm{min} / 1.73 \mathrm{~m}^{2}$ ) earlier than patients with CLDN16 mutations and have poorer renal survival [50].

There is no specific therapy for this syndrome. Patients can be treated by thiazide diuretics in order to reduce urinary $\mathrm{Ca}^{2+}$ excretion, potassium citrate (as citrate is a crystallization inhibitor) and $\mathrm{Mg}^{2+}$ supplements. Additionally, high fluid intake and salt restriction are advised. The effectiveness of these treatments on the course of nephrocalcinosis and on the decline of GFR in FHHNC is unknown. Hydrochlorothiazide has been shown to be effective in correcting hypercalciuria due to CLDN16 mutations in some [52,79] but not all patients [50-52]. $\mathrm{Mg}^{2+}$ supplementation might also be ineffective to correct hypomagnesemia [50-52]. Hydrochlorothiazide could aggravate hypomagnesemia. Treatment by amiloride can be discussed as potassium-sparing diuretics may have $\mathrm{Mg}^{2+}$-sparing properties [80].

Inhibitors of endocytosis may provide novel therapeutic strategies [81,82]. Blocking clathrin-mediated endocytosis increases surface expression of some CLDN16 mutants in vitro. Primaquine, an antimalarial agent increases also cell surface localization of a CLDN16 mutant in vitro [83].

The only treatment of end-stage renal disease is renal replacement therapy. As the primary defect resides in the kidney, there is no recurrence of FHHNC after kidney transplant.

\subsection{Variants/Pathogenesis}

To date, sixty-nine CLDN16 disease-causing variants have been described including missense/nonsense variants (53), splice site variants (5), small deletions (5), small insertions (2), small indels (2), gross deletions (1), complex mutation (1) (Table 4). 
Table 4. CLDN16 disease-causing variants [32].

\begin{tabular}{|c|c|c|c|c|c|c|}
\hline \multirow[b]{2}{*}{ Ref. } & \multicolumn{6}{|c|}{ Missense/Nonsense Mutations } \\
\hline & Nucleotide Change & Amino Acid Change & Protein Change & Variant Class & Exon & Domain \\
\hline [84] & c. $114 \mathrm{C}>\mathrm{A}$ & Cys38Term & p.C38* & $\mathrm{DM}^{\mathrm{a}}$ & 1 & $\mathrm{~N}$ term \\
\hline [78] & c. $211 \mathrm{~A}>\mathrm{G}$ & Met71Val & p.M71V & $\mathrm{DM}$ & 1 & $\mathrm{~N}$ term \\
\hline [26] & c. $212 \mathrm{~T}>\mathrm{G}$ & Met71Arg & p.M71R & $\mathrm{DM}$ & 1 & $\mathrm{~N}$ term \\
\hline [49] & c. $212 \mathrm{~T}>\mathrm{C}$ & Met71Thr & p.M71T & $\mathrm{DM}$ & 1 & $\mathrm{~N}$ term \\
\hline$[50]$ & c. $239 \mathrm{G}>\mathrm{A}$ & Cys80Tyr & p.C80Y & $\mathrm{DM}$ & 1 & TM1 \\
\hline [49] & c. $263 \mathrm{G}>\mathrm{A}$ & Gly88Glu & p.G88E & $\mathrm{DM}$ & 1 & TM1 \\
\hline$[85]$ & c. $290 \mathrm{~A}>\mathrm{G}$ & Asp97Gly & p.D97G & $\mathrm{DM}$ & 1 & ECS1 \\
\hline$[53]$ & c. $295 \mathrm{~T}>\mathrm{G}$ & Trp99Gly & p.W99G & DM & 1 & ECS1 \\
\hline$[49,52]$ & c. $330 \mathrm{C}>\mathrm{G}$ & Ser110Arg ${ }^{b}$ & p.S110R & $\mathrm{DM}$ & 2 & ECS1 \\
\hline$[49,86]$ & c. $341 \mathrm{G}>\mathrm{A}$ & Arg114Gln & p.R114Q & DM & 2 & ECS1 \\
\hline$[60]$ & c. $340 \mathrm{C}>\mathrm{T}$ & Arg114Term & p.R114* & $\mathrm{DM}$ & 2 & ECS1 \\
\hline$[56]$ & c. $346 \mathrm{C}>\mathrm{G}$ & Leu116Val & p.L116V & $\mathrm{DM}$ & 2 & ECS1 \\
\hline$[45,66]$ & c. $350 \mathrm{G}>\mathrm{A}$ & Trp117Term & p.W117* & $\mathrm{DM}$ & 2 & ECS1 \\
\hline [87] & c. $354 \mathrm{G}>\mathrm{A}$ & Trp118Term & p.W118* & $\mathrm{DM}$ & 2 & ECS1 \\
\hline$[52,88]$ & c. $358 \mathrm{~T}>\mathrm{C}$ & Cys120Arg & p.C120R & $\mathrm{DM}$ & 2 & ECS1 \\
\hline [49] & c. $385 \mathrm{C}>\mathrm{T}$ & Arg129Cys & p.R129C & $\mathrm{DM}$ & 2 & ECS1 \\
\hline$[50,54,64,71,86]$ & c. $416 \mathrm{C}>\mathrm{T}$ & Ala139Val & p.A139V & $\mathrm{DM}$ & 2 & ECS1 \\
\hline$[45,49,66,86]$ & c. $421 C>G$ & His141Asp & p.H141D & $\mathrm{DM}$ & 2 & ECS1 \\
\hline$[45-47,52,66,86]$ & c. $434 \mathrm{~T}>\mathrm{C}$ & Leu145Pro & p.L145P & $\mathrm{DM}$ & 3 & ECS1 \\
\hline$[86,89]$ & c. $446 \mathrm{G}>\mathrm{A}$ & Arg149Gln & p.R149Q & $\mathrm{DM}$ & 3 & ECS1 \\
\hline$[45,46,86]$ & c. $446 \mathrm{G}>\mathrm{T}$ & Arg149Leu & p.R149L & $\mathrm{DM}$ & 3 & ECS1 \\
\hline$[26,50]$ & c. $445 \mathrm{C}>\mathrm{T}$ & Arg149Term & p.R149* & $\mathrm{DM}$ & 3 & ECS1 \\
\hline$[45-47,49,52,66,86,88]$ & c. $453 \mathrm{G}>\mathrm{T}$ & Leu151Phe & p.L151F & $\mathrm{DM}$ & 3 & ECS1or TM2? \\
\hline$[45,49,66,86]$ & c. $452 \mathrm{~T}>\mathrm{G}$ & Leu151Trp & p.L151W & $\mathrm{DM}$ & 3 & ECS1or TM2? \\
\hline$[50,54,71,86]$ & c. $485 \mathrm{G}>\mathrm{T}$ & Gly162Val & p.G162V & $\mathrm{DM}$ & 3 & TM2 \\
\hline$[26,46]$ & c. $500 \mathrm{~T}>\mathrm{C}$ & Leu167Pro & p.L167P & $\mathrm{DM}$ & 3 & TM2 \\
\hline [90] & c. $539 \mathrm{C}>\mathrm{T}$ & Pro180Leu & p.P180L & $\mathrm{DM}$ & 3 & ICL \\
\hline$[50,87,91]$ & c. $547 \mathrm{~A}>\mathrm{G}$ & Lys183Glu & p.K183E & $\mathrm{DM}$ & 3 & ICL \\
\hline$[26,46,47,86]$ & c. $571 \mathrm{G}>\mathrm{A}$ & Gly191Arg & p.G191R & $\mathrm{DM}$ & 3 & TM3 \\
\hline [65] & c. $592 \mathrm{G}>\mathrm{C}$ & Gly198Arg & p.G198R & $\mathrm{DM}$ & 3 & TM3 \\
\hline$[45,86]$ & c. $593 \mathrm{G}>\mathrm{C}$ & Gly198Ala & p.G198A & DM & 4 & TM3 \\
\hline
\end{tabular}


Table 4. Cont.

\begin{tabular}{|c|c|c|c|c|c|c|}
\hline$[26,46,66,86]$ & c. $593 \mathrm{G}>\mathrm{A}$ & Gly198Asp & p.G198D & $\mathrm{DM}$ & 4 & TM3 \\
\hline$[63]$ & c. $602 \mathrm{G}>\mathrm{A}$ & Gly201Glu & p.G201E & $\mathrm{DM}$ & 4 & TM3 \\
\hline [92] & c. $620 \mathrm{G}>\mathrm{A}$ & Trp207Term & p.W207* & $\mathrm{DM}$ & 4 & ECS2 \\
\hline$[45-47,49]$ & c. $625 \mathrm{G}>\mathrm{A}$ & Ala209Thr & p.A209T & $\mathrm{DM}$ & 4 & ECS2 \\
\hline$[49,59,89]$ & c. $646 \mathrm{C}>\mathrm{T}$ & Arg216Cys & p.R216C & DM & 4 & ECS2 \\
\hline [55] & c. $647 \mathrm{G}>\mathrm{A}$ & Arg216His & p.R216H & $\mathrm{DM}$ & 4 & ECS2 \\
\hline$[49,93]$ & c. $679 \mathrm{G}>\mathrm{C}$ & Gly227Arg & p.G227R & $\mathrm{DM}$ & 4 & ECS2 \\
\hline$[26,46,47,94]$ & c. $695 \mathrm{~T}>\mathrm{G}$ & Phe232Cys & p.F232C & $\mathrm{DM}$ & 4 & ECS2 \\
\hline$[50]$ & c. $697 \mathrm{G}>\mathrm{C}$ & Gly233Arg & p.G233R & $\mathrm{DM}$ & 4 & ECS2 \\
\hline$[26,46]$ & c. $698 \mathrm{G}>\mathrm{A}$ & Gly233Asp & p.G233D & $\mathrm{DM}$ & 4 & ECS2 \\
\hline [61] & c. $697 \mathrm{G}>\mathrm{T}$ & Gly233Cys & p.G233C & $\mathrm{DM}$ & 4 & ECS2 \\
\hline [52] & c. $702 \mathrm{G}>\mathrm{T}$ & Trp234Cys & p.W234C & $\mathrm{DM}$ & 4 & ECS2 \\
\hline [26] & c. $704 \mathrm{C}>\mathrm{T}$ & Ser235Phe & p.S235F & $\mathrm{DM}$ & 4 & ECS2 \\
\hline$[45,46]$ & c.703T $>C$ & Ser235Pro & p.S235P & $\mathrm{DM}$ & 4 & ECS2 \\
\hline [77] & c. $704 \mathrm{C}>\mathrm{A}$ & Ser235Tyr & p.S235Y & $\mathrm{DM}$ & 4 & ECS2 \\
\hline$[49,95]$ & c. $710 \mathrm{G}>\mathrm{A}$ & Trp237Term & p.W237* & $\mathrm{DM}$ & 4 & ECS2 \\
\hline$[26,45,46,52,66,71,87]$ & c. $715 \mathrm{G}>\mathrm{A}$ & Gly239Arg & p.G239R & $\mathrm{DM}$ & 4 & ECL2 or TM4? \\
\hline [52] & c. $734 \mathrm{G}>\mathrm{A}$ & Gly245Asp & p.G245D & $\mathrm{DM}$ & 4 & TM4 \\
\hline [82] & c. $823 \mathrm{~A}>\mathrm{T}^{\mathrm{c}}$ & Lys275Term & p.K $275^{*}$ & $\mathrm{DM}$ & 5 & C term \\
\hline [81] & c. $831 \mathrm{~T}>\mathrm{G}^{\mathrm{d}}$ & Tyr277Term & p.Y277* & $\mathrm{DM}$ & 5 & C term \\
\hline [52] & c. $864 \mathrm{C}>\mathrm{G}$ & Tyr288Term & p.Y288* & $\mathrm{DM}$ & 5 & C term \\
\hline [96] & c. $908 \mathrm{C}>\mathrm{G}^{\mathrm{e}}$ & Thr303Arg & p.T303R & $\mathrm{DM}$ & 5 & C term \\
\hline \multicolumn{7}{|c|}{ Splicing Mutations } \\
\hline Ref. & Nucleotide Change & \multicolumn{2}{|c|}{ Splicing Mutation } & Variant Class & & \\
\hline [45] & c. $325-5 \mathrm{~T}>\mathrm{G}$ & \multicolumn{2}{|c|}{ IVS1 as T-G -5 } & $\mathrm{DM}$ & & \\
\hline$[60]$ & c. $427+5 \mathrm{G}>\mathrm{A}$ & \multicolumn{2}{|c|}{ IVS2 ds G-A +5 } & $\mathrm{DM}$ & & \\
\hline$[26]$ & c. $593-2 A>G$ & \multicolumn{2}{|c|}{ IVS3 as A-G -2 } & $\mathrm{DM}$ & & \\
\hline$[49,59]$ & c. $784+1 G>T$ & \multicolumn{2}{|c|}{ IVS4 ds G-T +1 } & $\mathrm{DM}$ & & \\
\hline$[45]$ & c.785-14T $>\mathrm{G}$ & \multicolumn{2}{|c|}{ IVS4 as T-G -14 } & $\mathrm{DM}$ & & \\
\hline
\end{tabular}


Table 4. Cont.

\begin{tabular}{|c|c|c|c|c|c|}
\hline & \multicolumn{5}{|c|}{ Small Deletions } \\
\hline & Nucleotide Change & Protein Change & Variant Class & Exon & Domain \\
\hline [97] & c.166delG ${ }^{f}$ & p.(Ala56Leufs*16) & DM? & 1 & $\mathrm{~N}$ term \\
\hline [49] & c. $235 \mathrm{delG} \mathrm{g}$ & p.(Ala79fsX90) & & 1 & TM1 \\
\hline [45] & c.368delA & p.(Asn123Metfs*21) & $\mathrm{DM}$ & 2 & ECS1 \\
\hline [49] & c.408_410delCAT & p.(Ile137del) & $\mathrm{DM}$ & 2 & ECS1 \\
\hline \multirow[t]{3}{*}{ [61] } & c.800delG & p.(Arg267Lysfs*7) & $\mathrm{DM}$ & 5 & C term \\
\hline & \multicolumn{5}{|c|}{ Small Insertions } \\
\hline & Nucleotide Change & Protein Change & Variant Class & Exon & Domain \\
\hline [74] & c.324+3_324+4insT & Not available & $\mathrm{DM}$ & intron 1 & \\
\hline \multirow[t]{3}{*}{ [71] } & c.545_548dupTTAA & p.(Lys183Asnfs*2) & $\mathrm{DM}$ & 3 & ICL \\
\hline & \multicolumn{5}{|c|}{ Small Indels } \\
\hline & Nucleotide Change & Protein Change & Variant Class & Exon & Domain \\
\hline$[45,66]$ & c.165_166delGGinsC & p.(Arg55Serfs*17) & $\mathrm{R}$ & 1 & $\mathrm{~N}$ term \\
\hline \multirow[t]{3}{*}[45,46]{} & c.646_647delCGinsAC & p.(Arg216Thr) & $\mathrm{DM}$ & 4 & ECS2 \\
\hline & \multicolumn{5}{|c|}{ Gross Deletions } \\
\hline & DNA Level & Description & Variant Class & Exon & Domain \\
\hline \multirow[t]{3}{*}[71,98]{} & g.DNA & Ex. 2-5 & $\mathrm{DM}$ & $2-5$ & \\
\hline & \multicolumn{5}{|c|}{ Complex Mutations } \\
\hline & & Description & Variant Class & Exon & Domain \\
\hline [62] & & c.574_589delins23bp & $\mathrm{DM}$ & 3 & TM3 \\
\hline
\end{tabular}

Variant class is described according the Human Gene Mutation Database [37] DM: Disease-causing mutations; DM?: probable/possible pathological mutation; «Retired records (R)», a variant that has been removed from HGMD if found to have been erroneously included ab initio or if the variant has been subject to retraction/correction in the literature resulting in the record becoming obsolete, merged or otherwise invalid. Domains are described according to authors and [99]. C Term, COOH terminus; TM, transmembrane domain; ECS1, first extracellular segment; ICL, intracellular loop; ECS2, second extracellular segment; $\mathrm{N}$ Term, $\mathrm{NH}_{2}$ terminus. ${ }^{a}$ : the "significance» described by Trujillano was "likely pathogenic according to ACMG guidelines." They categorized patients' phenotypes according to the Human Phenotype Ontology nomenclature based on the clinical data and preceding workup provided by the referring physician. The phenotype described was: psychosis, seizures, muscle weakness, respiratory failure, reduced dihydropyrimidine dehydrogenase activity, decreased body weight, reduced consciousness/confusion, lower limb muscle weakness; ${ }^{\text {b: }}$ reported as p.S110R 329AGC>AGG; ${ }^{\text {c: }}$ reported as p.L203*, c.822A $>$ T; ${ }^{\text {d. }}$ : reported as p.Y207*, c.620T>G; ${ }^{\text {e: }}$ reported as p.T233R, c.697C $>\mathrm{G}$; ${ }^{\mathrm{f}}$ : reported as c.164delG; ${ }^{\text {g: }}$ reported as $236 \mathrm{delG}$, p.A80fsX91 , ${ }^{*}$ indicates that the predicted consequence is a termination codon. 
CLDN16 mutations are located in the two ECSs but also affect the TMDs and the cytoplasmic regions. No link between the affected domain and the phenotype has been described.

The most frequent CLDN16 disease-causing variant, c.453G > T (p.L151F) is found in almost 50\% of the German and Eastern European patients described so far [45,52,100].

Twenty-two CLDN19 disease-causing variants have been described so far including missense/nonsense variants (19), small deletions (2), gross deletions (1) (Table 5). 
Table 5. CLDN19 disease-causing variants [32].

\begin{tabular}{|c|c|c|c|c|c|c|}
\hline \multirow[b]{2}{*}{ Ref. } & \multicolumn{6}{|c|}{ Missense/Nonsense Mutations } \\
\hline & Nucleotide Change & Amino Acid Change & Protein Change & Variant Class & Exon & Domain \\
\hline$[50]$ & c. $54 \mathrm{G}>\mathrm{A}$ & Trp18Term & p.W18* & $\mathrm{DM}$ & 1 & TM1 \\
\hline$[47,50,51,57,58,67,70,72,101-103]$ & c. $59 \mathrm{G}>\mathrm{A}^{\mathrm{a}, \mathrm{b}}$ & Gly20Asp & p.G20D & $\mathrm{DM}$ & 1 & TM1 \\
\hline$[50,101,102]$ & c. $83 \mathrm{C}>\mathrm{T}$ & Pro28Leu & p.P28L & $\mathrm{DM}$ & 1 & TM1 \\
\hline [51] & c. $122 \mathrm{~T}>\mathrm{C}$ & Ile41Thr & p.I41T & $\mathrm{DM}$ & 1 & ECS1 \\
\hline$[50,67]$ & c. $130 \mathrm{G}>\mathrm{A}$ & Val44Met & p.V44M & $\mathrm{DM}$ & 1 & ECS1 \\
\hline$[47,51,57]$ & c. $169 \mathrm{C}>\mathrm{G}$ & Gln57Glu & p.Q57E & $\mathrm{DM}$ & 1 & ECS1 \\
\hline$[50,72]$ & c. $169 \mathrm{C}>\mathrm{T}$ & Gln57Term & p.Q57* & $\mathrm{DM}$ & 1 & ECS1 \\
\hline [51] & c. $223 \mathrm{G}>\mathrm{T}$ & Gly75Cys & p.G75C & DM? & 1 & ECS1 \\
\hline [51] & c. $223 \mathrm{G}>\mathrm{A}$ & Gly75Ser & p.G75S & DM? & 1 & ECS1 \\
\hline$[68,76]^{\mathrm{c}}$ & c. $241 \mathrm{C}>\mathrm{T}$ & Arg81Trp & p.R81W & $\mathrm{DM}$ & 2 & ECS1 or TM2? \\
\hline [104] & c. $263 \mathrm{~T}>\mathrm{A}$ & Val88Glu & p.V88E & $\mathrm{DM}$ & 2 & TM2 \\
\hline$[72]$ & c. $269 \mathrm{~T}>\mathrm{G}$ & Leu90Arg & p.L90R & $\mathrm{DM}$ & 2 & TM2 \\
\hline$[47,57]$ & c. $269 \mathrm{~T}>\mathrm{C}$ & Leu90Pro & p.L90P & $\mathrm{DM}$ & 2 & TM2 \\
\hline [51] & c. $364 \mathrm{G}>\mathrm{A}$ & Gly122Arg & p.G122R & $\mathrm{DM}$ & 2 & TM3 \\
\hline [63] & c. $388 \mathrm{G}>\mathrm{T}$ & Gly130Asp & p.G130C & $\mathrm{DM}$ & 2 & TM3 \\
\hline$[63,75]$ & c. $389 \mathrm{G}>\mathrm{A}$ & Gly130Asp & p.G130D & $\mathrm{DM}$ & 3 & TM3 \\
\hline [105] & c. $506 \mathrm{G}>\mathrm{A}^{\mathrm{d}}$ & Trp169Term & p.W169* & $\mathrm{DM}$ & 4 & TM4 \\
\hline [94] & c. $535 \mathrm{G}>\mathrm{A}$ & Gly179Ser & p.G179S & $\mathrm{DM}$ & 4 & TM4 \\
\hline [72] & c. $599 \mathrm{G}>\mathrm{A}$ & Arg200Gln & p.R200Q & DM? & 4 & C term \\
\hline
\end{tabular}


Table 5. Cont.

\begin{tabular}{|c|c|c|c|c|c|c|}
\hline \multirow[b]{2}{*}{ Ref. } & \multicolumn{6}{|c|}{ Missense/Nonsense Mutations } \\
\hline & Nucleotide Change & Amino Acid Change & Protein Change & Variant Class & Exon & Domain \\
\hline & \multicolumn{6}{|c|}{ Small Deletions } \\
\hline & Nucleotide Change & \multicolumn{2}{|c|}{ Protein Change } & Variant Class & Exon & Domain \\
\hline [106] & c.140_141delAT & \multicolumn{2}{|c|}{ p. $\left(\right.$ Tyr $\left.47^{*}\right)$} & $\mathrm{DM}$ & 1 & ECS1 \\
\hline \multirow[t]{3}{*}{ [50] } & c.403_406delACTG & \multicolumn{2}{|c|}{ p.(Thr135Leuf*9) } & $\mathrm{DM}$ & 3 & TM3 \\
\hline & \multicolumn{6}{|c|}{ Gross Deletions } \\
\hline & DNA Level & Description & & Variant Class & Exon & Domain \\
\hline$[50]$ & g.DNA & Ex. 1-4 & & $\mathrm{DM}$ & $1-4$ & \\
\hline
\end{tabular}

Variant class is described according the Human Gene Mutation Database [37]. DM: Disease-causing mutations; DM?: probable/possible pathological mutation. Domains are described according to authors and [99]. C Term, COOH terminus; TM, transmembrane domain; ECS1, first extracellular segment; ICL, intracellular loop; ECS2, second extracellular segment; N Term, $\mathrm{NH}_{2}$ terminus. ${ }^{\text {a }}$ : reported as c.C>T in ref [58]; ${ }^{\text {; }}$ : reported as c.69G>A in ref [72]; ${ }^{c}$ : reported as p.Arg81Cys in ref [68]; ${ }^{\mathrm{d}}$ : reported as c.697G>A in ref [105]; ${ }^{*}$ indicates that the predicted consequence is a termination codon.. 
Most patients from Spain or southwestern France with CLDN19 mutation carry the CLDN19 p.(G20D) mutant that may reveal a founder effect $[50,51,57,103]$.

Cldn16 and Cldn19 are expressed at the tight junction of the C-TAL of Henle's loop in rodents and play a key role in the paracellular transport of $\mathrm{Ca}^{2+}$ and $\mathrm{Mg}^{2+}$. There, $25 \%$ of filtered $\mathrm{Ca}^{2+}$ and $70 \%$ of filtered $\mathrm{Mg}^{2+}$ are reabsorbed. The selective transport in the TAL of $\mathrm{Ca}^{2+}$ and of $\mathrm{Mg}^{2+}$ depends: (1) on a selective paracellular permeability to $\mathrm{Ca}^{2+}$ and $\mathrm{Mg}^{2+}$ and (2) on a driving force, the lumen-positive transepithelial voltage generated by the active transcellular transport of $\mathrm{NaCl}$ and at the end of the C-TAL by $\mathrm{NaCl}$ paracellular back flux as the epithelium is more permeable to $\mathrm{Na}^{+}$than to chloride [107] (Figure 2).

A

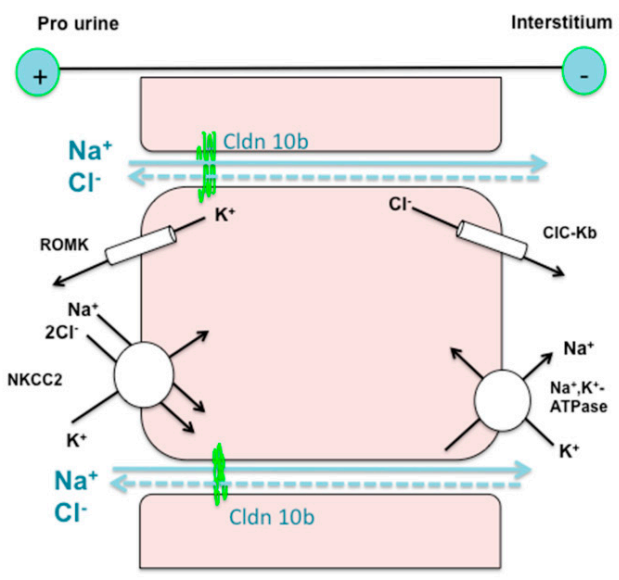

Inner Stripe of Outer Medulla Thick Ascending Limb
B

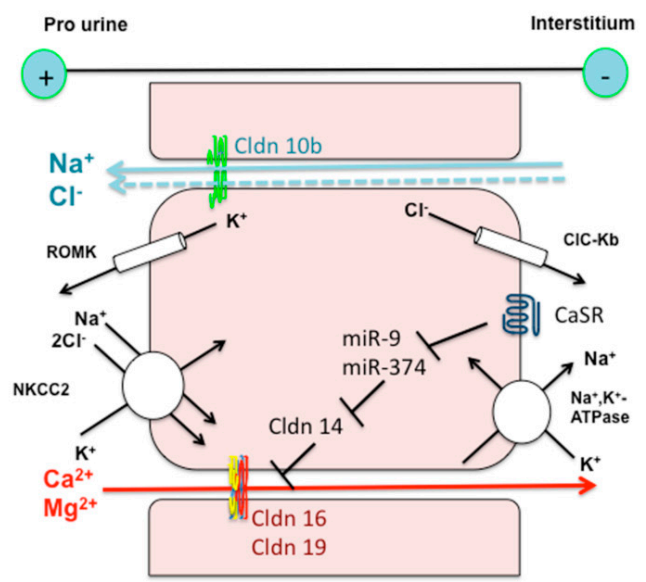

End of the cortical Thick Ascending Limb

\section{C}

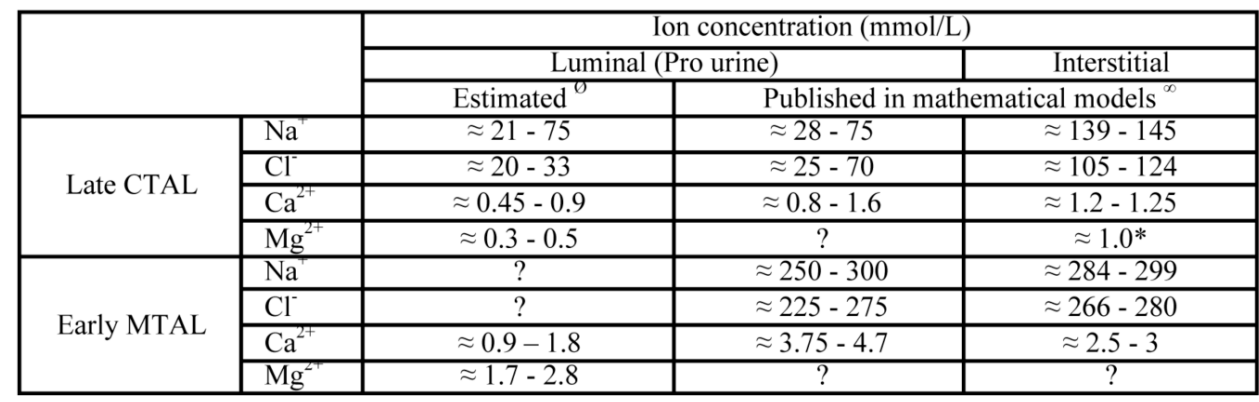

Figure 2. Model of ion transport in the inner stripe of outer medulla (IS-) (A) and in the cortical thick ascending limb of the loop of Henle (C-TAL) (B) and estimated ion concentration (C). The original works used to build the model have been published in References [108-110]. NaCl is reabsorbed via the apical cotransporter NKCC2. Most of the potassium that enters the cell recycles back to the lumen via the potassium channel ROMK, thereby hyperpolarizing the apical membrane, while most of the chloride leaves the cell across the basolateral chloride channel CLCKB, resulting in a depolarization of the membrane. Sodium exits the cell via the $\mathrm{Na}^{+}, \mathrm{K}^{+}$-ATPase at the basolateral membrane. The difference in voltage of the two membranes accounts for the lumen positive transepithelial potential difference, the driving force for the paracellular diffusion of divalent cations in the C-TAL and of sodium in the IS-TAL. Claudin (Cldn)16 and Cldn19 may confer a paracellular permeability and selectivity to cations. Cldn14 may interact with Cldn16 and inhibit the Cldn16/Cldn19 complex. It is suggested that Cldn10b drives paracellular $\mathrm{NaCl}$ back flux in cortical TAL, adding to the lumen-positive voltage as the paracellular pathway is more permeable to sodium than to chloride. High $\mathrm{Ca}^{2+}$ diet and allosteric agonists of calcium-sensing receptor (CaSR) may trigger the expression of Cldn14 via the inhibition of the transcription of two microRNAs miR-9 and miR-374 suppressing Cldn14 gene expression. ${ }^{\varnothing}$ Direct 
measurement of luminal ion concentration in the TAL is not possible because the segment is inaccessible to micropuncture. Luminal concentrations of $\mathrm{Na}^{+}$, chloride $\left(\mathrm{Cl}^{-}\right), \mathrm{Mg}^{2+}$ and $\mathrm{Ca}^{2+}$ in the early distal convoluted tubule, the segment just downstream the late cortical thick ascending limb (CTAL) have been measured during micropuncture experiments in rodents [111-119]. In most studies, concentrations are expressed as a ratio between tubular fluid and plasma ultrafilterable ion concentration. Plasma $\mathrm{NaCl}$ is freely filtered, whereas around $60 \%$ of $\mathrm{Ca}$ and $80 \%$ of $\mathrm{Mg}$ are ultrafilterable. Luminal ion concentrations in the early medullary thick ascending limb (MTAL) can be estimated based on a Ca ${ }^{2+}$ and $\mathrm{Mg}^{2+}$ reabsorption equaling $20-25 \%$ and $60-70 \%$ of filtered load, respectively and the lack of substantial water reabsorption in the TAL. ${ }^{\infty}$ Values of ion concentrations in the cortical interstitial fluid are similar to those in plasma, due to the dense capillary network and high blood flow in the cortex. Those concentrations have been reported in mathematical models except for magnesium $\left(^{*}\right)$. Estimates of the luminal and interstitial concentrations of $\mathrm{Na}^{+}, \mathrm{Cl}^{-}$and $\mathrm{Ca}^{2+}$ in the early medullary thick ascending limbs have been published in mathematical models [120-126].

The selective paracellular permeability to $\mathrm{Ca}^{2+}$ and $\mathrm{Mg}^{2+}$ is most likely conferred by the expression of Cldn16 and 19. In the TAL, Cldn3, 10b, 16 and 19 are expressed under basal conditions [127]. However TAL tight junctions show a mosaic expression of either Cldn10b or Cldn3/Cldn16/Cldn19 in the cortex and in the outer stripe of outer medulla (OS) in mice and rat [127] (Figure 3). Cldn16 is virtually absent from the inner stripe of outer medulla (IS) TAL whereas Cldn10b is highly expressed. Cldn19 is expressed in tight junction and detected intracellularly in OS- and C-TAL whereas it is only detected intracellularly in IS-TAL $[127,128]$. The Cldn16 proportion of total tight junction length in Cand OS-TAL ranges from 37 to $97 \%$ [127]. The permeability ratio $\mathrm{P}_{\mathrm{Mg}} / \mathrm{P}_{\mathrm{Na}}$ is higher in C- and OS-TAL than in IS-TAL [127].

A
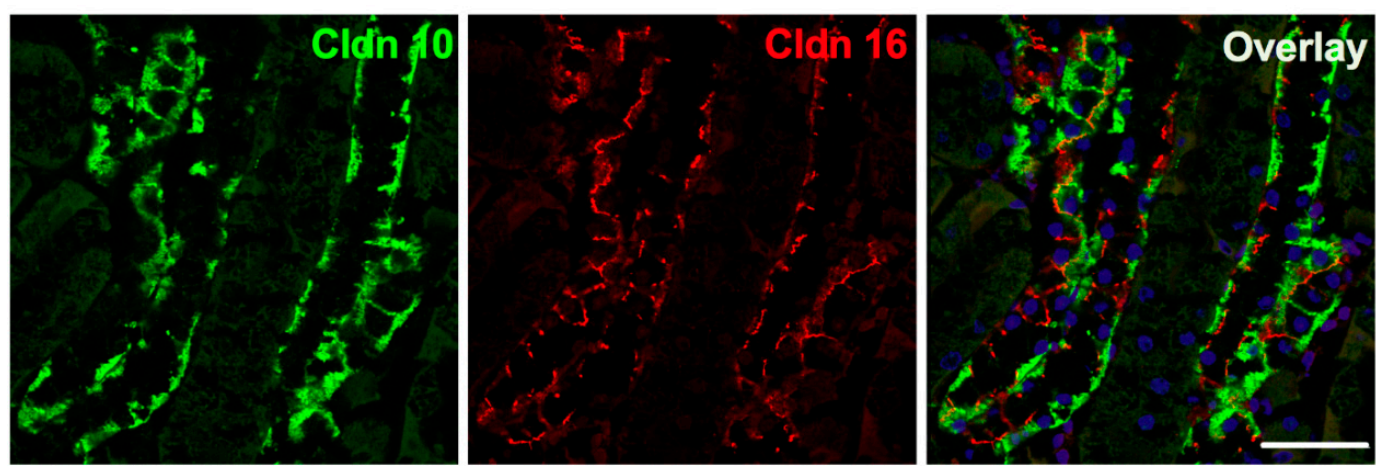

B
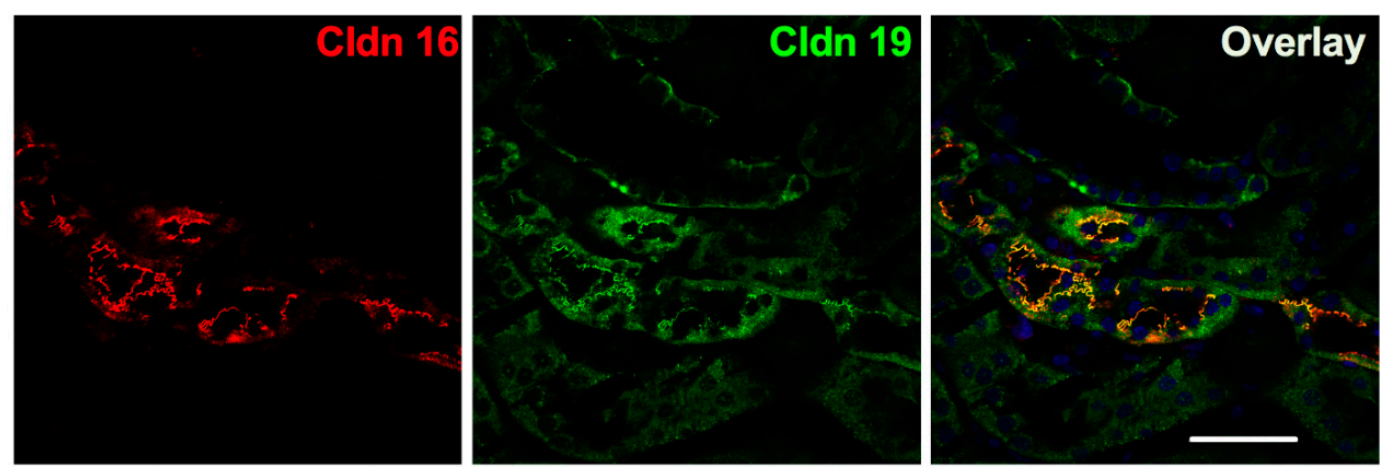

Figure 3. Expression of claudin 10, claudin 16 and claudin 19 in the murine cortical thick ascending limb in immunofluorescence. (A) Pattern of expression of claudin 10 and claudin 16 in the mouse C-TAL. Either claudin 10 (Cldn 10, in green) or claudin 16 (Cldn 16, in red) are expressed at tight junction. (B) Pattern of expression of claudin 16 and claudin 19 in the mouse C-TAL. Claudin 16 (Cldn 16 , in red) and claudin 19 (Cldn 19, in green) are colocalized at tight junction. Bar $=20 \mu \mathrm{m}$. 
In patients, bi allelic mutations of CLDN16 cause a selective defect in paracellular $\mathrm{Mg}^{2+}$ and $\mathrm{Ca}^{2+}$ reabsorption in the TAL, with intact $\mathrm{NaCl}$ reabsorption demonstrated by the normal response to furosemide infusion [54]. However the exact role of claudins 16 and 19 remain a matter of debate. In vitro permeability studies assessing the function of claudin 16 and/or claudin 19 expressed in cell lines have yielded contradicting results. Two hypotheses are been put forward. The first one is that claudin16/claudin19 might function as a divalent cation selective paracellular pore. The second is that claudin16/claudin19 increase $\mathrm{P}_{\mathrm{Na}} / \mathrm{P}_{\mathrm{Cl}}$, a prerequisite for the diffusion potential in the C-TAL.

Some investigators found that claudin 16 increases transepithelial transport of $\mathrm{Ca}^{2+}[129]$ and of $\mathrm{Mg}^{2+}[83,130,131]$, when heterogously expressed in epithelial cells (Table 6). Others found an effect of CLDN16 only on $\mathrm{Mg}^{2+}\left(\right.$ not $\left.\mathrm{Ca}^{2+}\right)$ permeability [48]. Still others found that CLDN16, when expressed in distinct cell lines, elicits only a small increase in transepithelial $\mathrm{Mg}^{2+}$ permeability but a greater increase in $\mathrm{Na}^{+}$permeability $[46,47]$. 
Table 6. Function of claudin 16 according to heterologous expression studies in cell lines.

\begin{tabular}{|c|c|c|c|c|c|c|c|c|c|c|c|}
\hline Claudin & Cell Line & Transfection & TER & $\mathbf{P}_{\mathrm{Na}} / \mathbf{P}_{\mathrm{Cl}}$ & $\mathbf{P}_{\mathrm{Na}}$ & $\mathbf{P}_{\mathrm{Cl}}$ & $\mathbf{P}_{\mathrm{Mg}}$ & $\mathbf{P}_{\mathrm{Ca}}$ & $\mathrm{Mg}^{2+}$ Flux & $\mathrm{Ca}^{2+}$ Flux & Ref. \\
\hline Rat Cldn16 & MDCK & stable & $\nearrow$ & & & & & & & $\nearrow^{*} \diamond \diamond$ & [129] \\
\hline Rat? Cldn16 & MDCK & stable & $\nearrow$ & $\searrow$ & $\searrow$ & NS & & & $\nearrow \S \varnothing$ & & [130] \\
\hline Rat Cldn16 & $\begin{array}{l}\text { MDCK } \\
\text { Tet-OFF }\end{array}$ & $\begin{array}{l}\text { Inducible } \\
\text { expression }\end{array}$ & $\nearrow$ & & & & & & $\nearrow \S^{*}$ & & [132] \\
\hline Human $\Delta 70$ CLDN16 & LLC-PK1 & Stable? & $\searrow$ & $\nearrow$ & $\nearrow$ & NS & $\nearrow^{+}$ & & & & [46] \\
\hline Human $\Delta 70$ CLDN16 & MDCK II & Stable? & & & NS & NS & NS & & & & [46] \\
\hline Full length Human CLDN16 & LLC-PK1 & Stable? & $\searrow$ & $\nearrow$ & $\nearrow$ & NS & & & & & [47] \\
\hline Full length Human CLDN16 & MDCK II & Stable? & & & NS & NS & & & & & [47] \\
\hline $\begin{array}{c}\text { Long version of human } \\
\text { CLDN16 }\end{array}$ & MDCK-C7 & Stable & & & NS & & $\nearrow^{\infty}$ & $\mathrm{NS}^{\infty}$ & & & [48] \\
\hline Full-length human CLDN16 & MDCK-C7 & stable & & NS & & & $\nearrow ¥$ & & & & [131] \\
\hline
\end{tabular}

Relative epithelial permeabilities (e.g., $\mathrm{P}_{\mathrm{Na}} / \mathrm{P}_{\mathrm{Cl}}$ ) are calculated using the Goldman-Hodgkin-Katz equation and the diffusion potential caused by the application of distinct solutions at the apical and basolateral compartment. Absolute permeabilities can be calculated if the transepithelial conductance is known using Kimizuka-Koketsu equation [41]. Measuring ion flux can be performed using either radioactive isotopes or fluorescent compounds or non-radioactive ions. Flux measurement include trans- and paracellular transport [41]. TER: trans epithelial resistance; NS: not significantly different from control; $\mathrm{P}_{\mathrm{x}}$ : permeability to ion $\mathrm{X} ;{ }^{\int}$ : Transepithelial transport of ${ }^{45} \mathrm{Ca}^{2+}$ measurement; ${ }^{\S}$ : The transepithelial transport of Mg ${ }^{2+}$ was measured using Xylidyl Blue-I.; ${ }^{+}$: The permeability of $\mathrm{Mg}^{2+}$ across monolayers was determined according to Tang and Goodenough [133]; ${ }^{£}: \mathrm{Mg}^{2+}$ flux was measured employing atomic absorption spectrometry; ${ }^{\infty}: \mathrm{Mg}^{2+}$ and $\mathrm{Ca}^{2+}$ permeabilities, calculated from dilution potential/bionic potential measurements; : Measurement of unidirectional fluxes from the basolateral to the apical side was performed under short-circuit conditions with $\mathrm{MgSO}_{4}$. The atomic absorption of $\mathrm{Mg}^{2+}$ was measured in an oxidizing air-acetylene flame at $285.2 \mathrm{~nm}$. $\mathrm{P}$ ( from resulting fluxes ( $\mathrm{P}_{\mathrm{Mg}}=$ flux/concentration); $\partial$ Permeability $\mathrm{PC}=$ Flux/Substrate concentration in cis compartment. This was then corrected for the permeability of blank filters, $\mathrm{PB}$, to obtain the true transepithelial permeability $(\mathrm{PT})$, using the following equation $\mathrm{PT}=[(1 / \mathrm{PC}) \&(1 / \mathrm{PB})]^{-1}$; ***: Increased basolateral $\mathrm{Mg}^{2+}$ concentration induces a short circuit current $(\mathrm{may}$ activate a transcellular $\mathrm{Cl}^{-}$current); ${ }^{*}$ : from apical to basal compartment, without affecting transport from basal to apical compartments; ${ }^{\varnothing}$ : from apical to basal compartment; ${ }^{\ominus}$ : The apical to basolateral flux was competitively inhibited by $\mathrm{Mg}^{2+}$. 
The mechanism by which claudin 19 affects $\mathrm{Mg}^{2+}$ and $\mathrm{Ca}^{2+}$ reabsorption is also unclear according to in vitro heterologous expression studies $[47,134]$ (Table 7).

Table 7. Function of claudin 19 and of claudin 16 and claudin 19 co-expression according to heterologous expression studies in cell lines.

\begin{tabular}{|c|c|c|c|c|c|c|c|c|c|}
\hline Claudin & Cell Line & Transfection & TER & $\mathbf{P}_{\mathrm{Na}} / \mathbf{P}_{\mathrm{Cl}}$ & $\mathbf{P}_{\mathrm{Na}}$ & $\mathbf{P}_{\mathrm{Cl}}$ & $P_{M g}$ & $P_{\mathrm{Ca}}$ & Ref. \\
\hline $\begin{array}{l}\text { Human } \\
\text { CLDN19 }\end{array}$ & LLC-PK1 & Stable? & $\nearrow$ & $\nearrow$ & NS & $\searrow$ & + & & [47] \\
\hline $\begin{array}{l}\text { Human } \\
\text { CLDN19 }\end{array}$ & MDCK II & Stable? & & & NS & NS & & & [47] \\
\hline $\begin{array}{l}\text { Mouse Cldn19 } \\
\text { Human }\end{array}$ & $\begin{array}{l}\text { MDCK II } \\
\text { Tet-Off cells }\end{array}$ & $\begin{array}{l}\text { Stable inducible } \\
\text { expression }\end{array}$ & $\nearrow$ & & $\searrow$ & NS & $\notin \partial े$ & $\searrow \partial$ & [134] \\
\hline $\begin{array}{l}\text { CLDN19 + full } \\
\text { length human } \\
\text { CLDN16 }\end{array}$ & LLC-PK1 & Stable? & $\searrow$ & $\nearrow$ & $\nearrow$ & $\searrow$ & $\searrow^{+}$ & & [47] \\
\hline
\end{tabular}

Relative epithelial permeabilities (e.g., $\mathrm{P}_{\mathrm{Na}} / \mathrm{P}_{\mathrm{Cl}}$ ) are calculated using the Goldman-Hodgkin-Katz equation and the diffusion potential caused by the application of distinct solutions at the apical and basolateral compartment. Absolute permeabilities can be calculated if the transepithelial conductance is known using Kimizuka-Koketsu equation [41]. TER: trans epithelial resistance; NS: not significantly different from control; $\mathrm{P}_{\mathrm{x}}$ : permeability to ion X; $\int$ : Transepithelial transport of ${ }^{45} \mathrm{Ca}^{2+}$ measurement; ${ }^{+}$: The permeability of $\mathrm{Mg}^{2+}$ across monolayers was determined according to Tang and Goodenough [133]; ${ }^{\ddagger}: \mathrm{Mg}^{2+}$ flux was measured employing atomic absorption spectrometry; $\partial$ : Permeability PC $=$ Flux/Substrate concentration in cis compartment. This was then corrected for the permeability of blank filters, $\mathrm{PB}$, to obtain the true transepithelial permeability $(\mathrm{PT})$, using the following equation $\mathrm{PT}=[(1 / \mathrm{PC}) \&$ $(1 / \mathrm{PB})]^{-1}$.

CLDN 16 and CLDN 19 can interact with each other [47], forming a cis heterodimer at the cell membrane in vitro [135]. Their co expression increases cation selectivity of the tight junction [47]: the effects of CLDN16 and of CLDN19, when co-expressed in LLC-PK1 cells, are synergistic on the $\mathrm{P}_{\mathrm{Na}} / \mathrm{P}_{\mathrm{Cl}}$ permeability ratio [47]: CLDN19 decreases the permeability to chloride whereas CLDN16 increases the permeability to $\mathrm{Na}^{+}$(Tables 5 and 6).

The functional consequences of some human CLDN16 mutations has also been studied by heterologous expression in cells in vitro. Disease-causing mutations can alter intracellular trafficking of claudin 16 or disturb claudin16-claudin 19 interaction or result in a loss of paracellular transport despite its expression at tight junction. Some mutations retain residual function whereas other result in a complete loss of function $[46,47,49,81,82,85,96,131]$. Similar results have been found with claudin 19 mutants [47,57].

Different mouse models have been engineered to better understand the pathophysiology of FHHNC. Cldn 16 knock-out (KO) model has been engineered a few years ago [136]. Cldn $16 \mathrm{KO}$ mice have hypomagnesemia and hypercalciuria [136]. Lack of Cldn16 decreases $\mathrm{P}_{\mathrm{Ca}} / \mathrm{P}_{\mathrm{Na}}$ and $\mathrm{P}_{\mathrm{Mg}} / \mathrm{P}_{\mathrm{Na}}$ in isolated, perfused CTAL whereas $\mathrm{P}_{\mathrm{Na}} / \mathrm{P}_{\mathrm{Cl}}$ is unaltered [43]. Cldn 16 knockdown (KD) and Cldn 19 KD mice models have been generated by RNA interference technology. Both have hypomagnesemia and significantly increased urinary excretion of $\mathrm{Ca}^{2+}$ without significant renal insufficiency [137-139]. $\mathrm{NaCl}$ absorption may be impaired as Cldn $16 \mathrm{KD}$ mice have low blood pressure and elevated plasma aldosterone concentration. In isolated, perfused TAL from Cldn $16 \mathrm{KD}, \mathrm{P}_{\mathrm{Na}} / \mathrm{P}_{\mathrm{Cl}}$ is decreased and $\mathrm{P}_{\mathrm{Na}} / \mathrm{P}_{\mathrm{Mg}}$ is unaffected [139]. Hou et al. conclude that Cldn16 is a non-specific cation channel [139]. The hypothesis is that the mutated Cldn16 protein has an indirect effect on $\mathrm{Mg}^{2+}$ reabsorption by decreasing $\mathrm{P}_{\mathrm{Na}} / \mathrm{P}_{\mathrm{Cl}}$, thereby reducing the diffusion potential/the transepithelial voltage and driving force for $\mathrm{Mg}^{2+}$ reabsorption $[138,139]$. These results are seemingly in conflict with those from Cldn10b cKO mice (see above). However, Cldn10b disruption also lowers $\mathrm{P}_{\mathrm{Na}} / \mathrm{P}_{\mathrm{Cl}}$ but affects IS-, OS- and C-TAL. In this model, the high transepithelial voltage may elevate passive paracellular $\mathrm{Mg}^{2+}$ and $\mathrm{Ca}^{2+}$ reabsorption in the C-TAL. Moreover, the mosaic pattern is abrogated and Cldn16 and Cldn19 are expressed in all tight junction in C- and OS- TAL and expression of Cldn 16 expands to IS- TAL, which likely increases the permeability to $\mathrm{Mg}^{2+}$ and $\mathrm{Ca}^{2+}[43,127]$. 
Of note, in mice models (Cldn10 KO, Cldn16 KO, Cldn10/Cldn16 double KO and Cldn $16 \mathrm{KD}$ ), bi-ionic diffusion potential may have been measured in the presence of high peritubular $\mathrm{MgCl}_{2}$ or $\mathrm{CaCl}_{2}$ concentration that activate the basolateral calcium-sensing receptor and make the interpretation of the data more complex $[39,43,139]$.

Renal abnormalities and electrolyte imbalances have not been investigated in Cldn19 KO mice [73].

Because of the described interaction between Cldn16 and Cldn19, one would have expected a loss of expression of Cldn19 in Cldn16 KO TAL. Surprisingly, the expression of Cldn19 is unaffected in Cldn16 KO mice. The mosaic pattern of expression of either Cldn10b or Cldn3/Cldn19 persisted [127]. In contrast, the majority of Cldn19 tight junctions immunostaining is lost in Cldn16 KD TAL and Cldn16 staining disappeared from tight junctions in Cldn19 KD TAL [137]. Whether the interaction of Cldn16 and Cldn19 is required for their assembly into tight junction is not clear in murine models. Unfortunately, no staining of renal biopsy from FHHNC patient has been performed to our best knowledge.

Renal tissue examination showed $\mathrm{Ca}^{2+}$ deposits along the basement membrane of medullary tubules of Cldn16 KD [139] but not of Cldn16 KO mice. None of the models faithfully recapitulate the human disease, they are not complicated by renal failure $[136,139]$. The pathogenesis of parenchymal deposition of $\mathrm{Ca}^{2+}$-containing crystal in the kidney (while $\mathrm{Ca}^{2+}$ is less reabsorbed) and of progressive renal failure in FHHNC remains unclear.

\section{Claudin 14}

The human CLDN14 gene, located on chromosome 21q22.3, contains 1 translated exon, encoding CLDN14, a protein of 239 amino acids [32].

Sixteen CLDN14 disease-causing variants have been described so far including missense/nonsense variants (12), regulatory substitutions (1) small deletions (2), small indels (1) (13 variants class «DM», 2 variants class «DM?», 1 variant class «Disease-associated polymorphisms with supporting functional evidence DFP») [32].

Mutations in CLDN14 cause autosomal recessive non-syndromic deafness-29 (DFNB29, OMIM \#614035) [140,141], a phenotype reproduced in Cldn14 KO mice [142]. Heterozygous mutations of CLDN14 have been described in neonates with vein of Galen malformation [143].

No rare variant of the CLDN14 gene has been described in Humans with abnormal renal ion handling. However, claudin 14 may have a role in renal $\mathrm{Ca}^{2+}$ and $\mathrm{Mg}^{2+}$ handling.

Claudin 14 may interact with claudin 16 in TAL and decrease the cation selectivity of the claudin 16-claudin 19 heteromeric complex [144].

The expression of Cldn14 is regulated by changes in dietary $\mathrm{Ca}^{2+}[144,145]$. Cldn14 is either not detected [144] or detected in few murine OS- and C-TAL with distinct location to the tight junction under control conditions; on a high $\mathrm{Ca}^{2+}$-containing diet, Cldn14 is highly expressed at murine tight junction of OS- and C-TAL but not in IS-TAL [128]. The expression of Cldn14 is also regulated by chronic changes in dietary $\mathrm{Mg}^{2+}$ content in mice and rat [146].

High $\mathrm{Ca}^{2+}$ diet and allosteric agonists of calcium-sensing receptor CaSR may trigger the expression of Cldn14 via the inhibition of the transcription of two microRNAs miR-9 and miR-374 suppressing Cldn14 gene expression [144,145,147].

Cldn14 KO mice have a higher plasma $\mathrm{Mg}^{2+}$ concentration with a lower fractional excretion rate of $\mathrm{Mg}^{2+}$ and of $\mathrm{Ca}^{2+}$ under high $\mathrm{Ca}^{2+}$ dietary condition [144]. On the other hand, overexpression of Cldn14 in TAL generates a phenotype with a lower plasma $\mathrm{Mg}^{2+}$ concentration and a higher fractional excretion rate of $\mathrm{Mg}^{2+}$ and of $\mathrm{Ca}^{2+}$ [147].

When overexpressed in cell culture models, Cldn 14 decreases cation permeability and $\mathrm{Ca}^{2+}$ flux $[142,145]$ (Table 8).

Some single-nucleotide polymorphisms (SNPs) of CLDN14 are associated with $24 \mathrm{~h}$ urinary $\mathrm{Ca}^{2+}$ excretion and/or kidney stones [148-153]. In silico analysis and in vitro studies of the SNP rs78250838:C $>$ T suggest that it may introduce a novel insulinoma-associated 1 (INSM1) transcription factor binding site, enhancing CLDN14 mRNA and protein expression [151]. Some of these SNPs 
are also associated with bone mineral density in women [148], serum total $\mathrm{CO}_{2}$ [148], $\mathrm{Mg}^{2+}$ [150], potassium [150] and PTH [148,150]. One the other hand, Corre et al. identified an SNP (rs172639) in a noncoding intergenic region that was associated with the urinary $\mathrm{Mg}^{2+}$ over $\mathrm{Ca}^{2+}$ concentration ratio in spot urine [146]. This SNP is part of a large linkage disequilibrium block spanning the 3' CLDN14 gene region that contains two microRNA (miR-374 and miR-9) binding sites [144,146].

Table 8. Function of claudin 14 according to heterologous expression studies in cell lines.

\begin{tabular}{|c|c|c|c|c|c|c|c|c|c|}
\hline Claudin & Cell Line & Transfection & TER & $\mathbf{P}_{\mathrm{Na}} / \mathbf{P}_{\mathrm{Cl}}$ & $\mathbf{P}_{\mathrm{Na}}$ & $\mathbf{P}_{\mathrm{Cl}}$ & $\mathbf{P}_{\mathrm{Ca}}$ & $\mathrm{Ca}^{2+}$ Flux & Ref. \\
\hline $\begin{array}{l}\text { Human } \\
\text { CLDN14 }\end{array}$ & $\begin{array}{l}\text { MDCK II } \\
\text { Tet-Off cells }\end{array}$ & $\begin{array}{l}\text { Stable inducible } \\
\text { expression }\end{array}$ & $\nearrow$ & $\searrow$ & $\searrow$ & & & & [142] \\
\hline $\begin{array}{l}\text { Mouse } \\
\text { Cldn14 }\end{array}$ & OK & Stable & $\nearrow$ & $\searrow$ & & NS & & $\searrow \int^{\varnothing}$ & [145] \\
\hline $\begin{array}{l}\text { Mouse } \\
\text { Cldn14 }\end{array}$ & $\begin{array}{l}\text { MDCK II } \\
\text { Tet-Off cells }\end{array}$ & $\begin{array}{l}\text { Stable inducible } \\
\text { expression }\end{array}$ & $\nearrow$ & $\searrow$ & $\searrow$ & NS & $\searrow$ & & [145] \\
\hline
\end{tabular}

Relative epithelial permeabilities (e.g., $\mathrm{P}_{\mathrm{Na}} / \mathrm{P}_{\mathrm{Cl}}$ ) are calculated using the Goldman-Hodgkin-Katz equation and the diffusion potential caused by the application of distinct solutions at the apical and basolateral compartment. Absolute permeabilities can be calculated if the transepithelial conductance is known using Kimizuka-Koketsu equation [41]. Flux measurement include trans- and paracellular transport [41]. TER: trans epithelial resistance; NS: not significantly different from control; $\mathrm{P}_{\mathrm{x}}$ : permeability to ion $\mathrm{X} ; \int$ : Transepithelial transport of ${ }^{45} \mathrm{Ca}^{2+}$ measurement; ${ }^{ø}$ : from apical to basal compartment.

\section{Conclusions}

Our knowledge regarding claudins in the mammalian kidney and their critical role in ion homeostasis has considerably expanded within the past two decades. This has largely been driven by the need to understand the clinical consequences and provide care to patients affected by rare syndromes caused by genetic mutations of some claudins. The severity of FHHNC and HELIX syndromes underscores the importance of paracellular transport in ion homeostasis. Despite the progresses made in the past years, many questions are left unanswered. Out of the many claudins expressed along the renal tubule and collecting duct, only three have been unequivocally traced to rare renal clinical syndromes in humans. It is quite possible that as yet unidentified syndromes will be recognized as been caused by rare variants of other claudins in the coming years. Besides, a lot has to be understood regarding the physiology of claudins. For example, we do not know which factors determine which claudin(s) is/are expressed at a given tight junction. This is particularly important in the C-TAL where one given cell can make tight junctions with its neighboring cells that differ in claudin composition. Although we know that the properties of tight junctions and hence that of claudins, are tightly regulated [154], we ignored most of the hormones and mechanisms that are involved in the short- and long-term control of claudin function and expression. Finally, providing a specific and effective treatment to patients with claudin-related rare diseases requires that we are able to target mutant claudin to the right tight junction and/or to correct the function of the mutant. The possibility to provide such treatment seems to be far away and will require substantial efforts. However, given the severity of claudin-related rare disease, it is worth the effort.

Author Contributions: C.P.-B. and P.H. have been equally involved in the drafting and the editing of the manuscript. Both approved the final version. C.P.-B. and P.H. agree to be personally accountable for the author's own contributions and for ensuring that questions related to the accuracy or integrity of any part of the work, even ones in which the author was not personally involved, are appropriately investigated, resolved and documented in the literature. All authors have read and agreed to the published version of the manuscript.

Funding: C.P.-B. is supported by the Fondation pour la Recherche Médicale (FRM FDT201904007918). This work was supported by grants from Agence Nationale de la Recherche (ANR-12-BSV1-0031-01 and ANR-17-CE14-0032 to P.H.).

Conflicts of Interest: C.P.-B. and P.H. have no conflict of interest to declare regarding the content of the present review. 


\section{Abbreviations}

$\begin{array}{ll}\text { CLDN } & \text { human claudin protein } \\ \text { CLDN } & \text { human claudin gene/mRNA } \\ \text { Cldn } & \text { rodent claudin protein } \\ \text { Cldn } & \text { rodent claudin gene/mRNA)) }\end{array}$

\section{References}

1. Pei, L.; Solis, G.; Nguyen, M.T.; Kamat, N.; Magenheimer, L.; Zhuo, M.; Li, J.; Curry, J.; McDonough, A.A.; Fields, T.A.; et al. Paracellular epithelial sodium transport maximizes energy efficiency in the kidney. J. Clin. Investig. 2016, 126, 2509-2518. [CrossRef] [PubMed]

2. Muto, S. Physiological roles of claudins in kidney tubule paracellular transport. Am. J. Physiol. Ren. Physiol. 2017, 312, F9-F24. [CrossRef] [PubMed]

3. Powell, D.W. Barrier function of epithelia. Am. J. Physiol. 1981, 241, G275-G288. [CrossRef] [PubMed]

4. Van Itallie, C.M.; Anderson, J.M. The molecular physiology of tight junction pores. Physiology 2004, 19, 331-338. [CrossRef] [PubMed]

5. Reuss, L. Tight junction permeability to ions and water. In Tight Junctions, 2nd ed.; Cereijido, M., Anderson, J.M., Eds.; CRC Press: Boca Raton, FL, USA, 2001; pp. 61-88.

6. Knipp, G.T.; Ho, N.F.; Barsuhn, C.L.; Borchardt, R.T. Paracellular diffusion in Caco-2 cell monolayers: Effect of perturbation on the transport of hydrophilic compounds that vary in charge and size. J. Pharm. Sci. 1997, 86, 1105-1110. [CrossRef] [PubMed]

7. Watson, C.J.; Rowland, M.; Warhurst, G. Functional modeling of tight junctions in intestinal cell monolayers using polyethylene glycol oligomers. Am. J. Physiol. Cell Physiol. 2001, 281, C388-C397. [CrossRef] [PubMed]

8. Saitoh, R.; Sugano, K.; Takata, N.; Tachibana, T.; Higashida, A.; Nabuchi, Y.; Aso, Y. Correction of permeability with pore radius of tight junctions in Caco-2 monolayers improves the prediction of the dose fraction of hydrophilic drugs absorbed by humans. Pharm. Res. 2004, 21, 749-755. [CrossRef]

9. Guo, P.; Weinstein, A.M.; Weinbaum, S. A dual-pathway ultrastructural model for the tight junction of rat proximal tubule epithelium. Am. J. Physiol. Ren. Physiol. 2003, 285, F241-F257. [CrossRef]

10. Yu, A.S.; Cheng, M.H.; Angelow, S.; Gunzel, D.; Kanzawa, S.A.; Schneeberger, E.E.; Fromm, M.; Coalson, R.D. Molecular basis for cation selectivity in claudin-2-based paracellular pores: Identification of an electrostatic interaction site. J. Gen. Physiol. 2009, 133, 111-127. [CrossRef]

11. Volkov, A.G.; Paula, S.; Deamer, D.W. Two mechanisms of permeation of small neutral molecules and hydrated ions across phospholipid bilayers. Bioelectrochem. Bioenerg. 1997, 42, 153-160. [CrossRef]

12. Chiba, H.; Osanai, M.; Murata, M.; Kojima, T.; Sawada, N. Transmembrane proteins of tight junctions. Biochim. Biophys. Acta 2008, 1778, 588-600. [CrossRef] [PubMed]

13. Furuse, M. Molecular basis of the core structure of tight junctions. Cold Spring Harb. Perspect. Biol. 2010, 2, a002907. [CrossRef] [PubMed]

14. Tsukita, S.; Furuse, M.; Itoh, M. Multifunctional strands in tight junctions. Nat. Rev. Mol. Cell Biol. 2001, 2, 285-293. [CrossRef] [PubMed]

15. Furuse, M.; Fujita, K.; Hiiragi, T.; Fujimoto, K.; Tsukita, S. Claudin-1 and -2: Novel integral membrane proteins localizing at tight junctions with no sequence similarity to occludin. J. Cell Biol. 1998, 141, 1539-1550. [CrossRef] [PubMed]

16. Morita, K.; Furuse, M.; Fujimoto, K.; Tsukita, S. Claudin multigene family encoding four-transmembrane domain protein components of tight junction strands. Proc. Natl. Acad. Sci. USA 1999, 96, 511-516. [CrossRef] [PubMed]

17. Tsukita, S.; Tanaka, H.; Tamura, A. The Claudins: From Tight Junctions to Biological Systems. Trends Biochem. Sci. 2019, 44, 141-152. [CrossRef]

18. Mineta, K.; Yamamoto, Y.; Yamazaki, Y.; Tanaka, H.; Tada, Y.; Saito, K.; Tamura, A.; Igarashi, M.; Endo, T.; Takeuchi, K.; et al. Predicted expansion of the claudin multigene family. FEBS Lett. 2011, 585, 606-612. [CrossRef]

19. Suzuki, H.; Nishizawa, T.; Tani, K.; Yamazaki, Y.; Tamura, A.; Ishitani, R.; Dohmae, N.; Tsukita, S.; Nureki, O.; Fujiyoshi, Y. Crystal structure of a claudin provides insight into the architecture of tight junctions. Science 2014, 344, 304-307. [CrossRef] 
20. Gong, Y.; Yu, M.; Yang, J.; Gonzales, E.; Perez, R.; Hou, M.; Tripathi, P.; Hering-Smith, K.S.; Hamm, L.L.; Hou, J. The Cap1-claudin-4 regulatory pathway is important for renal chloride reabsorption and blood pressure regulation. Proc. Natl. Acad. Sci. USA 2014, 111, E3766-E3774. [CrossRef]

21. Piontek, J.; Winkler, L.; Wolburg, H.; Muller, S.L.; Zuleger, N.; Piehl, C.; Wiesner, B.; Krause, G.; Blasig, I.E. Formation of tight junction: Determinants of homophilic interaction between classic claudins. FASEB J. 2008, 22, 146-158. [CrossRef]

22. Suzuki, H.; Tani, K.; Tamura, A.; Tsukita, S.; Fujiyoshi, Y. Model for the architecture of claudin-based paracellular ion channels through tight junctions. J. Mol. Biol. 2015, 427, 291-297. [CrossRef] [PubMed]

23. Alberini, G.; Benfenati, F.; Maragliano, L. A refined model of claudin-15 tight junction paracellular architecture by molecular dynamics simulations. PLoS ONE 2017, 12, e0184190. [CrossRef] [PubMed]

24. Itoh, M.; Sasaki, H.; Furuse, M.; Ozaki, H.; Kita, T.; Tsukita, S. Junctional adhesion molecule (JAM) binds to PAR-3: A possible mechanism for the recruitment of PAR-3 to tight junctions. J. Cell Biol. 2001, 154, 491-497. [CrossRef] [PubMed]

25. Itoh, M.; Furuse, M.; Morita, K.; Kubota, K.; Saitou, M.; Tsukita, S. Direct binding of three tight junction-associated MAGUKs, ZO-1, ZO-2, and ZO-3, with the COOH termini of claudins. J. Cell Biol. 1999, 147, 1351-1363. [CrossRef] [PubMed]

26. Simon, D.B.; Lu, Y.; Choate, K.A.; Velazquez, H.; Al-Sabban, E.; Praga, M.; Casari, G.; Bettinelli, A.; Colussi, G.; Rodriguez-Soriano, J.; et al. Paracellin-1, a renal tight junction protein required for paracellular $\mathrm{Mg}^{2+}$ resorption. Science 1999, 285, 103-106. [CrossRef]

27. Kirk, A.; Campbell, S.; Bass, P.; Mason, J.; Collins, J. Differential expression of claudin tight junction proteins in the human cortical nephron. Nephrol. Dial. Transplant. 2010, 25, 2107-2119. [CrossRef]

28. Hadj-Rabia, S.; Brideau, G.; Al-Sarraj, Y.; Maroun, R.C.; Figueres, M.L.; Leclerc-Mercier, S.; Olinger, E.; Baron, S.; Chaussain, C.; Nochy, D.; et al. Multiplex epithelium dysfunction due to CLDN10 mutation: The HELIX syndrome. Genet. Med. 2018, 20, 190-201. [CrossRef]

29. Krug, S.M.; Gunzel, D.; Conrad, M.P.; Rosenthal, R.; Fromm, A.; Amasheh, S.; Schulzke, J.D.; Fromm, M. Claudin-17 forms tight junction channels with distinct anion selectivity. Cell Mol. Life Sci. 2012, 69, 2765-2778. [CrossRef]

30. Gunzel, D.; Yu, A.S. Claudins and the modulation of tight junction permeability. Physiol. Rev. 2013, 93, 525-569. [CrossRef]

31. Yu, A.S. Claudins and the kidney. J. Am. Soc. Nephrol. 2015, 26, 11-19. [CrossRef]

32. HGMD Professional. 2019. Available online: http://www.hgmd.org/ (accessed on 12 December 2019).

33. Van Itallie, C.M.; Rogan, S.; Yu, A.; Vidal, L.S.; Holmes, J.; Anderson, J.M. Two splice variants of claudin-10 in the kidney create paracellular pores with different ion selectivities. Am. J. Physiol. Ren. Physiol. 2006, 291, F1288-F1299. [CrossRef] [PubMed]

34. Bongers, E.; Shelton, L.M.; Milatz, S.; Verkaart, S.; Bech, A.P.; Schoots, J.; Cornelissen, E.A.M.; Bleich, M.; Hoenderop, J.G.J.; Wetzels, J.F.M.; et al. A Novel Hypokalemic-Alkalotic Salt-Losing Tubulopathy in Patients with CLDN10 Mutations. J. Am. Soc. Nephrol. 2017, 28, 3118-3128. [CrossRef] [PubMed]

35. Klar, J.; Piontek, J.; Milatz, S.; Tariq, M.; Jameel, M.; Breiderhoff, T.; Schuster, J.; Fatima, A.; Asif, M.; Sher, M.; et al. Altered paracellular cation permeability due to a rare CLDN10B variant causes anhidrosis and kidney damage. PLoS Genet. 2017, 13, e1006897. [CrossRef] [PubMed]

36. Meyers, N.; Nelson-Williams, C.; Malaga-Dieguez, L.; Kaufmann, H.; Loring, E.; Knight, J.; Lifton, R.P.; Trachtman, H. Hypokalemia Associated With a Claudin 10 Mutation: A Case Report. Am. J. Kidney Dis. 2019, 73, 425-428. [CrossRef] [PubMed]

37. Stenson, P.D.; Mort, M.; Ball, E.V.; Evans, K.; Hayden, M.; Heywood, S.; Hussain, M.; Phillips, A.D.; Cooper, D.N. The Human Gene Mutation Database: Towards a comprehensive repository of inherited mutation data for medical research, genetic diagnosis and next-generation sequencing studies. Hum. Genet. 2017, 136, 665-677. [CrossRef]

38. Gunzel, D.; Stuiver, M.; Kausalya, P.J.; Haisch, L.; Krug, S.M.; Rosenthal, R.; Meij, I.C.; Hunziker, W.; Fromm, M.; Muller, D. Claudin-10 exists in six alternatively spliced isoforms that exhibit distinct localization and function. J. Cell Sci. 2009, 122, 1507-1517. [CrossRef] 
39. Breiderhoff, T.; Himmerkus, N.; Stuiver, M.; Mutig, K.; Will, C.; Meij, I.C.; Bachmann, S.; Bleich, M.; Willnow, T.E.; Muller, D. Deletion of claudin-10 (Cldn10) in the thick ascending limb impairs paracellular sodium permeability and leads to hypermagnesemia and nephrocalcinosis. Proc. Natl. Acad. Sci. USA 2012, 109, 14241-14246. [CrossRef]

40. Milatz, S.; Piontek, J.; Hempel, C.; Meoli, L.; Grohe, C.; Fromm, A.; Lee, I.M.; El-Athman, R.; Gunzel, D. Tight junction strand formation by claudin-10 isoforms and claudin-10a/-10b chimeras. Ann. NY Acad. Sci. 2017, 1405, 102-115. [CrossRef]

41. Gunzel, D.; Yu, A.S. Function and regulation of claudins in the thick ascending limb of Henle. Pflug. Arch. 2009, 458, 77-88. [CrossRef]

42. Angelow, S.; Schneeberger, E.E.; Yu, A.S. Claudin-8 expression in renal epithelial cells augments the paracellular barrier by replacing endogenous claudin-2. J. Membr. Biol. 2007, 215, 147-159. [CrossRef]

43. Breiderhoff, T.; Himmerkus, N.; Drewell, H.; Plain, A.; Gunzel, D.; Mutig, K.; Willnow, T.E.; Muller, D.; Bleich, M. Deletion of claudin-10 rescues claudin-16-deficient mice from hypomagnesemia and hypercalciuria. Kidney Int. 2018, 93, 580-588. [CrossRef] [PubMed]

44. Bourgeois, S.; Rossignol, P.; Grelac, F.; Chalumeau, C.; Klein, C.; Laghmani, K.; Chambrey, R.; Bruneval, P.; Duong, J.P.; Poggioli, J.; et al. Differentiated thick ascending limb (TAL) cultured cells derived from SV40 transgenic mice express functional apical NHE2 isoform: Effect of nitric oxide. Pflug. Arch. 2003, 446, 672-683. [CrossRef] [PubMed]

45. Weber, S.; Schneider, L.; Peters, M.; Misselwitz, J.; Ronnefarth, G.; Boswald, M.; Bonzel, K.E.; Seeman, T.; Sulakova, T.; Kuwertz-Broking, E.; et al. Novel paracellin-1 mutations in 25 families with familial hypomagnesemia with hypercalciuria and nephrocalcinosis. J. Am. Soc. Nephrol. 2001, 12, 1872-1881. [PubMed]

46. Hou, J.; Paul, D.L.; Goodenough, D.A. Paracellin-1 and the modulation of ion selectivity of tight junctions. J. Cell Sci. 2005, 118, 5109-5118. [CrossRef]

47. Hou, J.; Renigunta, A.; Konrad, M.; Gomes, A.S.; Schneeberger, E.E.; Paul, D.L.; Waldegger, S.; Goodenough, D.A. Claudin-16 and claudin-19 interact and form a cation-selective tight junction complex. J. Clin. Investig. 2008, 118, 619-628. [CrossRef]

48. Gunzel, D.; Amasheh, S.; Pfaffenbach, S.; Richter, J.F.; Kausalya, P.J.; Hunziker, W.; Fromm, M. Claudin-16 affects transcellular Cl-secretion in MDCK cells. J. Physiol. 2009, 587, 3777-3793. [CrossRef]

49. Konrad, M.; Hou, J.; Weber, S.; Dotsch, J.; Kari, J.A.; Seeman, T.; Kuwertz-Broking, E.; Peco-Antic, A.; Tasic, V.; Dittrich, K.; et al. CLDN16 genotype predicts renal decline in familial hypomagnesemia with hypercalciuria and nephrocalcinosis. J. Am. Soc. Nephrol. 2008, 19, 171-181. [CrossRef]

50. Godron, A.; Harambat, J.; Boccio, V.; Mensire, A.; May, A.; Rigothier, C.; Couzi, L.; Barrou, B.; Godin, M.; Chauveau, D.; et al. Familial hypomagnesemia with hypercalciuria and nephrocalcinosis: Phenotype-genotype correlation and outcome in 32 patients with CLDN16 or CLDN19 mutations. Clin. J. Am. Soc. Nephrol. 2012, 7, 801-809. [CrossRef]

51. Claverie-Martin, F.; Garcia-Nieto, V.; Loris, C.; Ariceta, G.; Nadal, I.; Espinosa, L.; Fernandez-Maseda, A.; Anton-Gamero, M.; Avila, A.; Madrid, A.; et al. Claudin-19 mutations and clinical phenotype in Spanish patients with familial hypomagnesemia with hypercalciuria and nephrocalcinosis. PLoS ONE 2013, 8, e53151. [CrossRef]

52. Sikora, P.; Zaniew, M.; Haisch, L.; Pulcer, B.; Szczepanska, M.; Moczulska, A.; Rogowska-Kalisz, A.; Bienias, B.; Tkaczyk, M.; Ostalska-Nowicka, D.; et al. Retrospective cohort study of familial hypomagnesaemia with hypercalciuria and nephrocalcinosis due to CLDN16 mutations. Nephrol. Dial. Transplant. 2015, 30, 636-644. [CrossRef]

53. Alparslan, C.; Oncel, E.P.; Akbay, S.; Alaygut, D.; Mutlubas, F.; Tatli, M.; Konrad, M.; Yavascan, O.; Kasap-Demir, B. A novel homozygous W99G mutation in CLDN-16 gene causing familial hypomagnesemic hypercalciuric nephrocalcinosis in Turkish siblings. Turk. J. Pediatrics 2018, 60, 76-80. [CrossRef] [PubMed]

54. Blanchard, A.; Jeunemaitre, X.; Coudol, P.; Dechaux, M.; Froissart, M.; May, A.; Demontis, R.; Fournier, A.; Paillard, M.; Houillier, P. Paracellin-1 is critical for magnesium and calcium reabsorption in the human thick ascending limb of Henle. Kidney Int. 2001, 59, 2206-2215. [CrossRef] [PubMed]

55. Deeb, A.; Abood, S.A.; Simon, J.; Dastoor, H.; Pearce, S.H.; Sayer, J.A. A novel CLDN16 mutation in a large family with familial hypomagnesaemia with hypercalciuria and nephrocalcinosis. BMC Res. Notes 2013, 6, 527. [CrossRef] [PubMed] 
56. Lu, J.; Zhao, X.; Paiardini, A.; Lang, Y.; Bottillo, I.; Shao, L. Familial hypomagnesaemia, Hypercalciuria and Nephrocalcinosis associated with a novel mutation of the highly conserved leucine residue 116 of Claudin 16 in a Chinese patient with a delayed diagnosis: A case report. BMC Nephrol. 2018, 19, 181. [CrossRef]

57. Konrad, M.; Schaller, A.; Seelow, D.; Pandey, A.V.; Waldegger, S.; Lesslauer, A.; Vitzthum, H.; Suzuki, Y.; Luk, J.M.; Becker, C.; et al. Mutations in the tight-junction gene claudin 19 (CLDN19) are associated with renal magnesium wasting, renal failure, and severe ocular involvement. Am. J. Hum. Genet. 2006, 79, 949-957. [CrossRef]

58. Arteaga, M.E.; Hunziker, W.; Teo, A.S.; Hillmer, A.M.; Mutchinick, O.M. Familial hypomagnesemia with hypercalciuria and nephrocalcinosis: Variable phenotypic expression in three affected sisters from Mexican ancestry. Ren. Fail. 2015, 37, 180-183. [CrossRef]

59. Hampson, G.; Konrad, M.A.; Scoble, J. Familial hypomagnesaemia with hypercalciuria and nephrocalcinosis (FHHNC): Compound heterozygous mutation in the claudin 16 (CLDN16) gene. BMC Nephrol. 2008, 9, 12. [CrossRef]

60. Hanssen, O.; Castermans, E.; Bovy, C.; Weekers, L.; Erpicum, P.; Dubois, B.; Bours, V.; Krzesinski, J.M.; Jouret, F. Two novel mutations of the CLDN16 gene cause familial hypomagnesaemia with hypercalciuria and nephrocalcinosis. Clin. Kidney J. 2014, 7, 282-285. [CrossRef]

61. Kang, J.H.; Choi, H.J.; Cho, H.Y.; Lee, J.H.; Ha, I.S.; Cheong, H.I.; Choi, Y. Familial hypomagnesemia with hypercalciuria and nephrocalcinosis associated with CLDN16 mutations. Pediatrics Nephrol. 2005, 20, 1490-1493. [CrossRef]

62. Lv, F.; Xu, X.J.; Wang, J.Y.; Liu, Y.; Jiang, Y.; Wang, O.; Xia, W.B.; Xing, X.P.; Li, M. A novel mutation in CLDN16 results in rare familial hypomagnesaemia with hypercalciuria and nephrocalcinosis in a Chinese family. Clin. Chim. Acta 2016, 457, 69-74. [CrossRef]

63. Perdomo-Ramirez, A.; Aguirre, M.; Davitaia, T.; Ariceta, G.; Ramos-Trujillo, E.; RenalTube, G.; Claverie-Martin, F. Characterization of two novel mutations in the claudin-16 and claudin-19 genes that cause familial hypomagnesemia with hypercalciuria and nephrocalcinosis. Gene 2019, 689, 227-234. [CrossRef] [PubMed]

64. Vargas-Poussou, R.; Cochat, P.; Le Pottier, N.; Roncelin, I.; Liutkus, A.; Blanchard, A.; Jeunemaitre, X. Report of a family with two different hereditary diseases leading to early nephrocalcinosis. Pediatrics Nephrol. 2008, 23, 149-153. [CrossRef] [PubMed]

65. Vianna, J.G.P.; Simor, T.G.; Senna, P.; De Bortoli, M.R.; Costalonga, E.F.; Seguro, A.C.; Luchi, W.M. Atypical presentation of familial hypomagnesemia with hypercalciuria and nephrocalcinosis in a patient with a new claudin-16 gene mutation. Clin. Nephrol. Case Stud. 2019, 7, 27-34. [CrossRef] [PubMed]

66. Weber, S.; Hoffmann, K.; Jeck, N.; Saar, K.; Boeswald, M.; Kuwertz-Broeking, E.; Meij, I.I.; Knoers, N.V.; Cochat, P.; Sulakova, T.; et al. Familial hypomagnesaemia with hypercalciuria and nephrocalcinosis maps to chromosome 3q27 and is associated with mutations in the PCLN-1 gene. Eur. J. Hum. Genet. 2000, 8, 414-422. [CrossRef]

67. Faguer, S.; Chauveau, D.; Cintas, P.; Tack, I.; Cointault, O.; Rostaing, L.; Vargas-Poussou, R.; Ribes, D. Renal, ocular, and neuromuscular involvements in patients with CLDN19 mutations. Clin. J. Am. Soc. Nephrol. 2011, 6, 355-360. [CrossRef]

68. Al-Shibli, A.; Konrad, M.; Altay, W.; Al Masri, O.; Al-Gazali, L.; Al Attrach, I. Familial hypomagnesemia with hypercalciuria and nephrocalcinosis (FHHNC): Report of three cases with a novel mutation in CLDN19 gene. Saudi J. Kidney Dis. Transplant. 2013, 24, 338-344. [CrossRef]

69. Peng, S.; Rao, V.S.; Adelman, R.A.; Rizzolo, L.J. Claudin-19 and the barrier properties of the human retinal pigment epithelium. Investig. Ophthalmol. Vis. Sci. 2011, 52, 1392-1403. [CrossRef]

70. Wang, S.B.; Xu, T.; Peng, S.; Singh, D.; Ghiassi-Nejad, M.; Adelman, R.A.; Rizzolo, L.J. Disease-associated mutations of claudin-19 disrupt retinal neurogenesis and visual function. Commun. Biol. 2019, 2, 113. [CrossRef]

71. Bardet, C.; Courson, F.; Wu, Y.; Khaddam, M.; Salmon, B.; Ribes, S.; Thumfart, J.; Yamaguti, P.M.; Rochefort, G.Y.; Figueres, M.L.; et al. Claudin-16 Deficiency Impairs Tight Junction Function in Ameloblasts, Leading to Abnormal Enamel Formation. J. Bone Miner. Res. 2016, 31, 498-513. [CrossRef] 
72. Yamaguti, P.M.; Neves, F.A.; Hotton, D.; Bardet, C.; de La Dure-Molla, M.; Castro, L.C.; Scher, M.D.; Barbosa, M.E.; Ditsch, C.; Fricain, J.C.; et al. Amelogenesis imperfecta in familial hypomagnesaemia and hypercalciuria with nephrocalcinosis caused by CLDN19 gene mutations. J. Med. Genet. 2017, 54, $26-37$. [CrossRef]

73. Miyamoto, T.; Morita, K.; Takemoto, D.; Takeuchi, K.; Kitano, Y.; Miyakawa, T.; Nakayama, K.; Okamura, Y.; Sasaki, H.; Miyachi, Y.; et al. Tight junctions in Schwann cells of peripheral myelinated axons: A lesson from claudin-19-deficient mice. J. Cell Biol. 2005, 169, 527-538. [CrossRef] [PubMed]

74. Nadarajah, L.; Khosravi, M.; Dumitriu, S.; Klootwijk, E.; Kleta, R.; Yaqoob, M.M.; Walsh, S.B. A novel claudin-16 mutation, severe bone disease, and nephrocalcinosis. Lancet 2014, 383, 98. [CrossRef]

75. Naeem, M.; Hussain, S.; Akhtar, N. Mutation in the tight-junction gene claudin 19 (CLDN19) and familial hypomagnesemia, hypercalciuria, nephrocalcinosis (FHHNC) and severe ocular disease. Am. J. Nephrol. 2011, 34, 241-248. [CrossRef] [PubMed]

76. Yuan, T.; Pang, Q.; Xing, X.; Wang, X.; Li, Y.; Li, J.; Wu, X.; Li, M.; Wang, O.; Jiang, Y.; et al. First report of a novel missense CLDN19 mutations causing familial hypomagnesemia with hypercalciuria and nephrocalcinosis in a Chinese family. Calcif. Tissue Int. 2015, 96, 265-273. [CrossRef]

77. Sanjad, S.A.; Hariri, A.; Habbal, Z.M.; Lifton, R.P. A novel PCLN-1 gene mutation in familial hypomagnesemia with hypercalciuria and atypical phenotype. Pediatrics Nephrol. 2007, 22, 503-508. [CrossRef]

78. Seeley, H.H.; Loomba-Albrecht, L.A.; Nagel, M.; Butani, L.; Bremer, A.A. Familial hypomagnesemia with hypercalciuria and nephrocalcinosis in three siblings having the same genetic lesion but different clinical presentations. World J. Pediatrics 2012, 8, 177-180. [CrossRef]

79. Zimmermann, B.; Plank, C.; Konrad, M.; Stohr, W.; Gravou-Apostolatou, C.; Rascher, W.; Dotsch, J. Hydrochlorothiazide in CLDN16 mutation. Nephrol. Dial. Transplant. 2006, 21, 2127-2132. [CrossRef]

80. Alexander, R.T.; Dimke, H. Effect of diuretics on renal tubular transport of calcium and magnesium. Am. J. Physiol. Ren. Physiol. 2017, 312, F998-F1015. [CrossRef]

81. Muller, D.; Kausalya, P.J.; Bockenhauer, D.; Thumfart, J.; Meij, I.C.; Dillon, M.J.; van't Hoff, W.; Hunziker, W. Unusual clinical presentation and possible rescue of a novel claudin-16 mutation. J. Clin. Endocrinol. Metab. 2006, 91, 3076-3079. [CrossRef]

82. Muller, D.; Kausalya, P.J.; Meij, I.C.; Hunziker, W. Familial hypomagnesemia with hypercalciuria and nephrocalcinosis: Blocking endocytosis restores surface expression of a novel Claudin-16 mutant that lacks the entire C-terminal cytosolic tail. Hum. Mol. Genet. 2006, 15, 1049-1058. [CrossRef]

83. Marunaka, K.; Fujii, N.; Kimura, T.; Furuta, T.; Hasegawa, H.; Matsunaga, T.; Endo, S.; Ikari, A. Rescue of tight junctional localization of a claudin-16 mutant D97S by antimalarial medicine primaquine in Madin-Darby canine kidney cells. Sci. Rep. 2019, 9, 9647. [CrossRef] [PubMed]

84. Trujillano, D.; Bertoli-Avella, A.M.; Kumar Kandaswamy, K.; Weiss, M.E.; Koster, J.; Marais, A.; Paknia, O.; Schroder, R.; Garcia-Aznar, J.M.; Werber, M.; et al. Clinical exome sequencing: Results from 2819 samples reflecting 1000 families. Eur. J. Hum. Genet. 2017, 25, 176-182. [CrossRef] [PubMed]

85. Guran, T.; Akcay, T.; Bereket, A.; Atay, Z.; Turan, S.; Haisch, L.; Konrad, M.; Schlingmann, K.P. Clinical and molecular characterization of Turkish patients with familial hypomagnesaemia: Novel mutations in TRPM6 and CLDN16 genes. Nephrol. Dial. Transplant. 2012, 27, 667-673. [CrossRef] [PubMed]

86. Perdomo-Ramirez, A.; de Armas-Ortiz, M.; Ramos-Trujillo, E.; Suarez-Artiles, L.; Claverie-Martin, F. Exonic CLDN16 mutations associated with familial hypomagnesemia with hypercalciuria and nephrocalcinosis can induce deleterious mRNA alterations. BMC Med. Genet. 2019, 20, 6. [CrossRef] [PubMed]

87. Braun, D.A.; Schueler, M.; Halbritter, J.; Gee, H.Y.; Porath, J.D.; Lawson, J.A.; Airik, R.; Shril, S.; Allen, S.J.; Stein, D.; et al. Whole exome sequencing identifies causative mutations in the majority of consanguineous or familial cases with childhood-onset increased renal echogenicity. Kidney Int. 2016, 89, 468-475. [CrossRef] [PubMed]

88. Staiger, K.; Staiger, H.; Haas, C.; Thamer, C.; Risler, T.; Machicao, F.; Haring, H.U. Hypomagnesemia and nephrocalcinosis in a patient with two heterozygous mutations in the CLDN16 gene. J. Nephrol. 2007, 20, 107-110. [PubMed]

89. Tajima, T.; Nakae, J.; Fujieda, K. Two heterozygous mutations of CLDN16 in a Japanese patient with FHHNC. Pediatrics Nephrol. 2003, 18, 1280-1282. [CrossRef] 
90. Yavarna, T.; Al-Dewik, N.; Al-Mureikhi, M.; Ali, R.; Al-Mesaifri, F.; Mahmoud, L.; Shahbeck, N.; Lakhani, S.; AlMulla, M.; Nawaz, Z.; et al. High diagnostic yield of clinical exome sequencing in Middle Eastern patients with Mendelian disorders. Hum. Genet. 2015, 134, 967-980. [CrossRef]

91. Kasapkara, C.S.; Tumer, L.; Okur, I.; Hasanoglu, A. A novel mutation of the claudin 16 gene in familial hypomagnesemia with hypercalciuria and nephrocalcinosis mimicking rickets. Genet. Couns. 2011, 22, 187-192.

92. Margabandhu, S.; Doshi, M. Familial Hypomagnesemia, Hypercalciuria and Nephrocalcinosis with Novel Mutation. Indian J. Nephrol. 2019, 29, 57-61. [CrossRef]

93. Kutluturk, F.; Temel, B.; Uslu, B.; Aral, F.; Azezli, A.; Orhan, Y.; Konrad, M.; Ozbey, N. An unusual patient with hypercalciuria, recurrent nephrolithiasis, hypomagnesemia and G227R mutation of Paracellin-1. An unusual patient with hypercalciuria and hypomagnesemia unresponsive to thiazide diuretics. Horm. Res. 2006, 66, 175-181. [PubMed]

94. Daga, A.; Majmundar, A.J.; Braun, D.A.; Gee, H.Y.; Lawson, J.A.; Shril, S.; Jobst-Schwan, T.; Vivante, A.; Schapiro, D.; Tan, W.; et al. Whole exome sequencing frequently detects a monogenic cause in early onset nephrolithiasis and nephrocalcinosis. Kidney Int. 2018, 93, 204-213. [CrossRef] [PubMed]

95. Turkmen, M.; Kasap, B.; Soylu, A.; Bober, E.; Konrad, M.; Kavukcu, S. Paracellin-1 gene mutation with multiple congenital abnormalities. Pediatrics Nephrol. 2006, 21, 1776-1778. [CrossRef] [PubMed]

96. Muller, D.; Kausalya, P.J.; Claverie-Martin, F.; Meij, I.C.; Eggert, P.; Garcia-Nieto, V.; Hunziker, W. A novel claudin 16 mutation associated with childhood hypercalciuria abolishes binding to ZO-1 and results in lysosomal mistargeting. Am. J. Hum. Genet. 2003, 73, 1293-1301. [CrossRef]

97. Zhou, F.; Mao, J.; Ye, Q.; Zhu, X.; Zhang, Y.; Ye, Y.; Fu, H.; Shen, H.; Lu, Z.; Xia, Y.; et al. Clinical features and genetic findings in Chinese children with distal renal tubular acidosis. Int. J. Clin. Exp. Pathol. 2018, 11, 3523-3532.

98. Yamaguti, P.M.; dos Santos, P.A.; Leal, B.S.; Santana, V.B.; Mazzeu, J.F.; Acevedo, A.C.; Neves Fde, A. Identification of the first large deletion in the CLDN16 gene in a patient with FHHNC and late-onset of chronic kidney disease: Case report. BMC Nephrol. 2015, 16, 92. [CrossRef]

99. Uniprot. Available online: https://www.uniprot.org (accessed on 20 December 2019).

100. Claverie-Martin, F.; Vargas-Poussou, R.; Muller, D.; Garcia-Nieto, V. Clinical utility gene card for: Familial hypomagnesemia with hypercalciuria and nephrocalcinosis with/without severe ocular involvement. Eur. J. Hum. Genet. 2015, 23. [CrossRef]

101. Almeida, J.R.; Machado Gde, A.; dos Santos, M.M.; Lopes Pde, F.; de Matos, J.P.; Neves, A.C.; Lugon, J.R. Five years results after intrafamilial kidney post-transplant in a case of familial hypomagnesemia due to a claudin-19 mutation. J. Bras. Nefrol. 2014, 36, 401-405. [CrossRef]

102. Haisch, L.; Almeida, J.R.; Abreu da Silva, P.R.; Schlingmann, K.P.; Konrad, M. The role of tight junctions in paracellular ion transport in the renal tubule: Lessons learned from a rare inherited tubular disorder. Am. J. Kidney Dis. 2011, 57, 320-330. [CrossRef]

103. Martin-Nunez, E.; Cordoba-Lanus, E.; Gonzalez-Acosta, H.; Oliet, A.; Izquierdo, E.; Claverie-Martin, F. Haplotype analysis of CLDN19 single nucleotide polymorphisms in Spanish patients with familial hypomagnesemia with hypercalciuria and nephrocalcinosis. World J. Pediatrics 2015, 11, 272-275. [CrossRef]

104. Khan, A.O.; Patel, N.; Ghazi, N.G.; Alzahrani, S.S.; Arold, S.T.; Alkuraya, F.S. Familial non-syndromic macular pseudocoloboma secondary to homozygous CLDN19 mutation. Ophthalmic Genet. 2018, 39, 577-583. [CrossRef] [PubMed]

105. Ekinci, Z.; Karabas, L.; Konrad, M. Hypomagnesemia-hypercalciuria-nephrocalcinosis and ocular findings: A new claudin-19 mutation. Turk. J. Pediatrics 2012, 54, 168-170.

106. Sharma, S.; Place, E.; Lord, K.; Leroy, B.P.; Falk, M.J.; Pradhan, M. Claudin 19-based familial hypomagnesemia with hypercalciuria and nephrocalcinosis in a sibling pair. Clin. Nephrol. 2016, 85, 346-352. [CrossRef] [PubMed]

107. Dimke, H.; Schnermann, J. Axial and cellular heterogeneity in electrolyte transport pathways along the thick ascending limb. Acta Physiol. 2018, 223, e13057. [CrossRef] [PubMed]

108. Greger, R. Chloride reabsorption in the rabbit cortical thick ascending limb of the loop of Henle. A sodium dependent process. Pflug. Arch. 1981, 390, 38-43. [CrossRef] 
109. Greger, R.; Schlatter, E. Properties of the basolateral membrane of the cortical thick ascending limb of Henle's loop of rabbit kidney. A model for secondary active chloride transport. Pflug. Arch. 1983, 396, 325-334. [CrossRef]

110. Greger, R.; Schlatter, E. Properties of the lumen membrane of the cortical thick ascending limb of Henle's loop of rabbit kidney. Pflug. Arch. 1983, 396, 315-324. [CrossRef]

111. Carney, S.L.; Wong, N.L.; Quamme, G.A.; Dirks, J.H. Effect of magnesium deficiency on renal magnesium and calcium transport in the rat. J. Clin. Investig. 1980, 65, 180-188. [CrossRef]

112. Le Grimellec, C.; Roinel, N.; Morel, F. Simultaneous Mg, Ca, P,K,Na and $\mathrm{Cl}$ analysis in rat tubular fluid. I. During perfusion of either inulin or ferrocyanide. Pflug. Arch. 1973, 340, 181-196. [CrossRef]

113. Costanzo, L.S.; Windhager, E.E. Calcium and sodium transport by the distal convoluted tubule of the rat. Am. J. Physiol. 1978, 235, F492-F506. [CrossRef]

114. Edwards, B.R.; Baer, P.G.; Sutton, R.A.; Dirks, J.H. Micropuncture study of diuretic effects on sodium and calcium reabsorption in the dog nephron. J. Clin. Investig. 1973, 52, 2418-2427. [CrossRef] [PubMed]

115. Stumpe, K.O.; Lowitz, H.D.; Ochwadt, B. Fluid reabsorption in Henle'sloop and urinary excretion of sodium and water in normal rats and rats with chronic hypertension. J. Clin. Investig. 1970, 49, 1200-1212. [CrossRef] [PubMed]

116. Vallon, V.; Richter, K.; Blantz, R.C.; Thomson, S.; Osswald, H. Glomerular hyperfiltration in experimental diabetes mellitus: Potential role of tubular reabsorption. J. Am. Soc. Nephrol. 1999, 10, 2569-2576. [PubMed]

117. Vallon, V.; Osswald, H.; Blantz, R.C.; Thomson, S. Potential role of luminal potassium in tubuloglomerular feedback. J. Am. Soc. Nephrol. 1997, 8, 1831-1837.

118. Schnermann, J.; Briggs, J.; Schubert, G. In situ studies of the distal convoluted tubule in the rat. I. Evidence for $\mathrm{NaCl}$ secretion. Am. J. Physiol. 1982, 243, F160-F166. [CrossRef]

119. Luke, R.G.; Wright, F.S.; Fowler, N.; Kashgarian, M.; Giebisch, G.H. Effects of potassium depletion on renal tubular chloride transport in the rat. Kidney Int. 1978, 14, 414-427. [CrossRef]

120. Edwards, A.; Castrop, H.; Laghmani, K.; Vallon, V.; Layton, A.T. Effects of NKCC2 isoform regulation on $\mathrm{NaCl}$ transport in thick ascending limb and macula densa: A modeling study. Am. J. Physiol. Ren. Physiol. 2014, 307, F137-F146. [CrossRef]

121. Edwards, A. Regulation of calcium reabsorption along the rat nephron: A modeling study. Am. J. Physiol. Ren. Physiol. 2015, 308, F553-F566. [CrossRef]

122. Layton, A.T.; Vallon, V.; Edwards, A. A computational model for simulating solute transport and oxygen consumption along the nephrons. Am. J. Physiol. Ren. Physiol. 2016, 311, F1378-F1390. [CrossRef]

123. Tournus, M.; Seguin, N.; Perthame, B.; Thomas, S.R.; Edwards, A. A model of calcium transport along the rat nephron. Am. J. Physiol. Ren. Physiol. 2013, 305, F979-F994. [CrossRef]

124. Nieves-Gonzalez, A.; Clausen, C.; Layton, A.T.; Layton, H.E.; Moore, L.C. Transport efficiency and workload distribution in a mathematical model of the thick ascending limb. Am. J. Physiol. Ren. Physiol. 2013, 304, F653-F664. [CrossRef] [PubMed]

125. Weinstein, A.M. A mathematical model of rat ascending Henle limb. III. Tubular function. Am. J. Physiol Ren. Physiol 2010, 298, F543-F556. [CrossRef] [PubMed]

126. Weinstein, A.M. A mathematical model of rat proximal tubule and loop of Henle. Am. J. Physiol. Ren. Physiol. 2015, 308, F1076-F1097. [CrossRef] [PubMed]

127. Milatz, S.; Himmerkus, N.; Wulfmeyer, V.C.; Drewell, H.; Mutig, K.; Hou, J.; Breiderhoff, T.; Muller, D.; Fromm, M.; Bleich, M.; et al. Mosaic expression of claudins in thick ascending limbs of Henle results in spatial separation of paracellular $\mathrm{Na}+$ and $\mathrm{Mg}^{2+}$ transport. Proc. Natl. Acad. Sci. USA 2017, 114, E219-E227. [CrossRef] [PubMed]

128. Plain, A.; Wulfmeyer, V.C.; Milatz, S.; Klietz, A.; Hou, J.; Bleich, M.; Himmerkus, N. Corticomedullary difference in the effects of dietary $\mathrm{Ca}^{2+}$ on tight junction properties in thick ascending limbs of Henle's loop. Pflug. Arch. 2016, 468, 293-303. [CrossRef] [PubMed]

129. Ikari, A.; Hirai, N.; Shiroma, M.; Harada, H.; Sakai, H.; Hayashi, H.; Suzuki, Y.; Degawa, M.; Takagi, K. Association of paracellin-1 with ZO-1 augments the reabsorption of divalent cations in renal epithelial cells. J. Biol. Chem. 2004, 279, 54826-54832. [CrossRef]

130. Ikari, A.; Matsumoto, S.; Harada, H.; Takagi, K.; Hayashi, H.; Suzuki, Y.; Degawa, M.; Miwa, M. Phosphorylation of paracellin-1 at Ser217 by protein kinase A is essential for localization in tight junctions. J. Cell Sci. 2006, 119, 1781-1789. [CrossRef] 
131. Kausalya, P.J.; Amasheh, S.; Gunzel, D.; Wurps, H.; Muller, D.; Fromm, M.; Hunziker, W. Disease-associated mutations affect intracellular traffic and paracellular $\mathrm{Mg}^{2+}$ transport function of Claudin-16. J. Clin. Investig. 2006, 116, 878-891. [CrossRef]

132. Ikari, A.; Kinjo, K.; Atomi, K.; Sasaki, Y.; Yamazaki, Y.; Sugatani, J. Extracellular Mg(2+) regulates the tight junctional localization of claudin-16 mediated by ERK-dependent phosphorylation. Biochim. Biophys. Acta 2010, 1798, 415-421. [CrossRef]

133. Tang, V.W.; Goodenough, D.A. Paracellular ion channel at the tight junction. Biophys. J. 2003, 84, 1660-1673. [CrossRef]

134. Angelow, S.; El-Husseini, R.; Kanzawa, S.A.; Yu, A.S. Renal localization and function of the tight junction protein, claudin-19. Am. J. Physiol. Ren. Physiol. 2007, 293, F166-F177. [CrossRef] [PubMed]

135. Gong, Y.; Renigunta, V.; Zhou, Y.; Sunq, A.; Wang, J.; Yang, J.; Renigunta, A.; Baker, L.A.; Hou, J. Biochemical and biophysical analyses of tight junction permeability made of claudin-16 and claudin-19 dimerization. Mol. Biol. Cell 2015, 26, 4333-4346. [CrossRef] [PubMed]

136. Will, C.; Breiderhoff, T.; Thumfart, J.; Stuiver, M.; Kopplin, K.; Sommer, K.; Gunzel, D.; Querfeld, U.; Meij, I.C.; Shan, Q.; et al. Targeted deletion of murine Cldn16 identifies extra- and intrarenal compensatory mechanisms of $\mathrm{Ca}^{2+}$ and $\mathrm{Mg}^{2+}$ wasting. Am. J. Physiol. Ren. Physiol. 2010, 298, F1152-F1161. [CrossRef] [PubMed]

137. Hou, J.; Renigunta, A.; Gomes, A.S.; Hou, M.; Paul, D.L.; Waldegger, S.; Goodenough, D.A. Claudin-16 and claudin-19 interaction is required for their assembly into tight junctions and for renal reabsorption of magnesium. Proc. Natl. Acad. Sci. USA 2009, 106, 15350-15355. [CrossRef]

138. Himmerkus, N.; Shan, Q.; Goerke, B.; Hou, J.; Goodenough, D.A.; Bleich, M. Salt and acid-base metabolism in claudin-16 knockdown mice: Impact for the pathophysiology of FHHNC patients. Am. J. Physiol. Ren. Physiol. 2008, 295, F1641-F1647. [CrossRef]

139. Hou, J.; Shan, Q.; Wang, T.; Gomes, A.S.; Yan, Q.; Paul, D.L.; Bleich, M.; Goodenough, D.A. Transgenic RNAi depletion of claudin-16 and the renal handling of magnesium. J. Biol. Chem. 2007, 282, 17114-17122. [CrossRef]

140. Wilcox, E.R.; Burton, Q.L.; Naz, S.; Riazuddin, S.; Smith, T.N.; Ploplis, B.; Belyantseva, I.; Ben-Yosef, T.; Liburd, N.A.; Morell, R.J.; et al. Mutations in the gene encoding tight junction claudin-14 cause autosomal recessive deafness DFNB29. Cell 2001, 104, 165-172. [CrossRef]

141. Bashir, Z.E.; Latief, N.; Belyantseva, I.A.; Iqbal, F.; Riazuddin, S.A.; Khan, S.N.; Friedman, T.B.; Riazuddin, S.; Riazuddin, S. Phenotypic variability of CLDN14 mutations causing DFNB29 hearing loss in the Pakistani population. J. Hum. Genet. 2013, 58, 102-108. [CrossRef]

142. Ben-Yosef, T.; Belyantseva, I.A.; Saunders, T.L.; Hughes, E.D.; Kawamoto, K.; Van Itallie, C.M.; Beyer, L.A.; Halsey, K.; Gardner, D.J.; Wilcox, E.R.; et al. Claudin 14 knockout mice, a model for autosomal recessive deafness DFNB29, are deaf due to cochlear hair cell degeneration. Hum. Mol. Genet. 2003, 12, 2049-2061. [CrossRef]

143. Duran, D.; Zeng, X.; Jin, S.C.; Choi, J.; Nelson-Williams, C.; Yatsula, B.; Gaillard, J.; Furey, C.G.; Lu, Q.; Timberlake, A.T.; et al. Mutations in Chromatin Modifier and Ephrin Signaling Genes in Vein of Galen Malformation. Neuron 2019, 101, 429-443 e424. [CrossRef]

144. Gong, Y.; Renigunta, V.; Himmerkus, N.; Zhang, J.; Renigunta, A.; Bleich, M.; Hou, J. Claudin-14 regulates renal $\mathrm{Ca}(+)(+)$ transport in response to CaSR signalling via a novel microRNA pathway. EMBO J. 2012, 31, 1999-2012. [CrossRef] [PubMed]

145. Dimke, H.; Desai, P.; Borovac, J.; Lau, A.; Pan, W.; Alexander, R.T. Activation of the $\mathrm{Ca}^{2+}$-sensing receptor increases renal claudin-14 expression and urinary $\mathrm{Ca}^{2+}$ excretion. Am. J. Physiol. Ren. Physiol. 2013, 304, F761-F769. [CrossRef] [PubMed]

146. Corre, T.; Olinger, E.; Harris, S.E.; Traglia, M.; Ulivi, S.; Lenarduzzi, S.; Belge, H.; Youhanna, S.; Tokonami, N.; Bonny, O.; et al. Common variants in CLDN14 are associated with differential excretion of magnesium over calcium in urine. Pflugers Arch. 2017, 469, 91-103. [CrossRef] [PubMed]

147. Gong, Y.; Hou, J. Claudin-14 underlies $\mathrm{Ca}(+)(+)$-sensing receptor-mediated $\mathrm{Ca}(+)(+)$ metabolism via NFAT-microRNA-based mechanisms. J. Am. Soc. Nephrol. 2014, 25, 745-760. [CrossRef]

148. Thorleifsson, G.; Holm, H.; Edvardsson, V.; Walters, G.B.; Styrkarsdottir, U.; Gudbjartsson, D.F.; Sulem, P.; Halldorsson, B.V.; de Vegt, F.; d'Ancona, F.C.; et al. Sequence variants in the CLDN14 gene associate with kidney stones and bone mineral density. Nat. Genet. 2009, 41, 926-930. [CrossRef] 
149. Guha, M.; Bankura, B.; Ghosh, S.; Pattanayak, A.K.; Ghosh, S.; Pal, D.K.; Puri, A.; Kundu, A.K.; Das, M. Polymorphisms in CaSR and CLDN14 Genes Associated with Increased Risk of Kidney Stone Disease in Patients from the Eastern Part of India. PLoS ONE 2015, 10, e0130790. [CrossRef]

150. Oddsson, A.; Sulem, P.; Helgason, H.; Edvardsson, V.O.; Thorleifsson, G.; Sveinbjornsson, G.; Haraldsdottir, E.; Eyjolfsson, G.I.; Sigurdardottir, O.; Olafsson, I.; et al. Common and rare variants associated with kidney stones and biochemical traits. Nat. Commun. 2015, 6, 7975. [CrossRef]

151. Ure, M.E.; Heydari, E.; Pan, W.; Ramesh, A.; Rehman, S.; Morgan, C.; Pinsk, M.; Erickson, R.; Herrmann, J.M.; Dimke, H.; et al. A variant in a cis-regulatory element enhances claudin-14 expression and is associated with pediatric-onset hypercalciuria and kidney stones. Hum. Mutat. 2017, 38, 649-657. [CrossRef]

152. Toka, H.R.; Genovese, G.; Mount, D.B.; Pollak, M.R.; Curhan, G.C. Frequency of rare allelic variation in candidate genes among individuals with low and high urinary calcium excretion. PLOS ONE 2013, 8, e71885. [CrossRef]

153. Arcidiacono, T.; Simonini, M.; Lanzani, C.; Citterio, L.; Salvi, E.; Barlassina, C.; Spotti, D.; Cusi, D.; Manunta, P.; Vezzoli, G. Claudin-14 Gene Polymorphisms and Urine Calcium Excretion. Clin. J. Am. Soc. Nephrol. 2018, 13, 1542-1549. [CrossRef]

154. Loupy, A.; Ramakrishnan, S.K.; Wootla, B.; Chambrey, R.; de la Faille, R.; Bourgeois, S.; Bruneval, P.; Mandet, C.; Christensen, E.I.; Faure, H.; et al. PTH-independent regulation of blood calcium concentration by the calcium-sensing receptor. J. Clin. Investig. 2012, 122, 3355-3367. [CrossRef] [PubMed]

(C) 2020 by the authors. Licensee MDPI, Basel, Switzerland. This article is an open access article distributed under the terms and conditions of the Creative Commons Attribution (CC BY) license (http://creativecommons.org/licenses/by/4.0/). 